\title{
The amino acid/polyamine/organocation (APC) superfamily of transporters specific for amino acids, polyamines and organocations
}

\author{
Donald L. Jack, Ian T. Paulsen† and Milton H. Saier, Jr \\ Author for correspondence: Milton H. Saier, Jr. Tel: +1 858534 4084. Fax: +1 8585347108. \\ e-mail:msaier@ucsd.edu
}

Department of Biology, University of California at San Diego, La Jolla, CA 92093-0116, USA

\begin{abstract}
In this paper an analysis of $\mathbf{1 7 5}$ currently sequenced transport proteins that comprise the amino acid/polyamine/organocation (APC) superfamily is reported. Members of this superfamily fall into 10 well-defined families that are either prokaryote specific, eukaryote specific or ubiquitous. Most of these proteins exhibit 12 probable transmembrane spanners (TMSs), but members of two of these families deviate from this pattern, exhibiting 10 and 14 TMSs. All members of these families are tabulated, their functional properties are reviewed and phylogenetic/sequence analyses define the evolutionary relationships of the proteins to each other. Evidence is presented that the APC superfamily may include two other currently recognized families that exhibit greater degrees of sequence divergence from APC superfamily members than do the proteins of the 10 established families from each other. At least some of the protein members of these two distantly related families exhibit 11 established TMSs. Altogether, the APC superfamily probably includes 12 currently recognized families with members that exhibit exclusive specificity for amino acids and their derivatives but which can possess 10, 11, 12 or 14 TMSs per polypeptide chain.
\end{abstract}

Keywords : amino acids, transport, evolution, superfamily, secondary carriers

\section{INTRODUCTION}

During the early evolution of life on Earth, before archaea and eukarya diverged from bacteria, life forms were undoubtedly much simpler than they are now. The primordial prokaryotic cell probably possessed few transporters and these served the essential functions of maintaining cytoplasmic ionic homeostasis and transmembrane electrical potentials, allowing entry and exit of plentiful nutrients by facilitation, and allowing the accumulation of scarce nutrients employing energy-

\footnotetext{
† Present address: The Institute for Genomic Research (TIGR), 9712 Medical Center Drive, Rockville, MD 20850, USA.

Abbreviations: AAAP, amino acid/auxin permease; AAT, amino acid transporter; $A B T$, archaeal/bacterial transporter; $A C T$, amino acid/choline transporter; APA, basic amino acid/polyamine antiporter; APC, amino acid/polyamine/organocation; CAT, cationic amino acid transporter; EAT, ethanolamine transporter; GGA, glutamate:GABA antiporter; HAAAP, hydroxy/aromatic amino acid permease; LAT, L-type amino acid transporter; SGP, spore germination protein; TC, transporter classification; TMS, transmembrane $\alpha$-helical spanner; YAT, yeast amino acid transporter.
}

coupled transporters. These early transport systems provided essential 'housekeeping' functions without which the cells could not survive. Due to gene duplication and divergence as well as speciation, these early transporters developed into our current, large, ubiquitous superfamilies.

One of the half-dozen largest of these superfamilies is the amino acid/polyamine/organocation (APC) superfamily [TC (transporter classification) no. 2.A.3; Closs et al., 1993; Reizer et al., 1993; Saier, 1998, 1999a, b, c, 2000a, b; see also http://www-biology.ucsd.edu/ $\sim$ msaier/transport/]. This superfamily includes members that function as solute: cation symporters and solute: solute antiporters (Devés \& Boyd, 1998; Isnard et al., 1996; Kashiwagi et al., 1997). Evidence suggests that in mammalian cells, simultaneous co- and countertransport of ions with the amino acid can occur with a variety of complicated stoichiometries. For example, uptake of an amino acid can be accompanied by cotransport of three $\mathrm{Na}^{+}$and one $\mathrm{Cl}^{-}$while being countertransported against a $\mathrm{K}^{+}$(Beckman \& Quick, 1998). 
The majority of homologous integral membrane APC transport proteins appear to exhibit a uniform topology with twelve transmembrane $\alpha$-helical spanners (TMSs) in a single polypeptide. This predicted topology has been experimentally verified for several bacterial homologues (Cosgriff \& Pittard, 1997; Ellis et al., 1995; Hu \& King, 1998a). APC permease polypeptide chains vary in size from about 400 aminoacyl residues to about 800 residues (Sophianopoulou \& Diallinas, 1995). The larger proteins exhibit hydrophilic N- and C-terminal extensions as well as occasional enlarged inter-TMS loops. Proteins of one group, the LAT (L-type amino acid transporter) family (TC no. 2.A.3.8) derived from animals, are sometimes found in association with auxiliary proteins of the rBAT family (TC no. 8.A.9), and some of these same proteins, when defective, give rise to human diseases (Devés \& Boyd, 1998; Estévez et al., 1998; Markovich et al., 1993; Mastroberardino et al., 1998; Palacín et al., 1998; Sato et al., 1999; Torrents et al., 1998; Verrey et al., 1999). Some of the animal homologues of the CAT (cationic amino acid transporter) family (TC no. 2.A.3.3) have been found to serve as viral receptors (Kim et al., 1991; Reizer et al., 1993; Wang et al., 1991).

The substrate specificities of several APC superfamily permeases have been carefully studied revealing that while some have exceptionally broad specificity, others are restricted to just one or a few amino acids or related compounds (Brechtel \& King, 1998; Hu \& King, 1998b, c; Isnard et al., 1996; Kashiwagi et al., 1997; Sato et al., 1999). One of these permeases, a histidine permease of Saccharomyces cerevisiae, surprisingly, has been implicated in manganese transport (Farcasanu et al., 1998). Their regulatory properties have been the subject of investigations (see, for example, Sanders et al., 1998).

No comprehensive phylogenetic analysis of the APC superfamily has been conducted since 1993, when only 14 members of the family were analysed (Reizer et al., 1993). Six years later, this superfamily has expanded to include 175 sequenced members that cluster in 10 defined families. Representation is found in all major domains of life (Paulsen et al., 1998a, b and unpublished results; see http://www-biology.ucsd.edu/ ipaulsen/ transport/). A recent study has led to the suggestion that this superfamily is related to other currently recognized families of amino acid transporters, but this suggestion has yet to be rigorously established (Young et al., 1999).

In this paper we update our earlier reports, providing structural, functional and phylogenetic descriptions of the proteins of the APC superfamily. All currently sequenced members of each of the 10 families that comprise this superfamily are tabulated, and their substrate specificities and modes of action, when known, are described. Phylogenetic tree construction reveals their evolutionary relationships to each other. Motif analyses provide sequence fingerprints for each of the 10 constituent families of the APC superfamily. The members of each of these families are also shown to exhibit uniform or nearly uniform topological features that prove characteristic of the superfamily as a whole.
Distinctive properties that distinguish some of the different families are also noted. Thus, while all members of eight families appear to exhibit 12 TMSs, the members of one prokaryotic family [the SGP (spore germination protein) family] have 10 TMSs while the eukaryotic members of one of the ubiquitous families (the CAT family) have 14 TMSs. Further evidence is presented for a relationship of the APC superfamily to two other families of amino acid transporters.

\section{METHODS}

Computer methods. Sequences of the proteins that comprise the 10 families within the APC superfamily were obtained by recursive PSI-BLAST searches without iteration until all potential members had been retrieved from the GenBank, PIR and SWISS-PROT databases $\left(P\right.$ value $\geqslant 10^{-4}$ ) (Altschul et al., 1997). Phylogenetic trees were constructed based on multiple alignments developed with the CLUSTAL x 8.1 (Thompson et al., 1997) and the TREe (Feng \& Doolittle, 1990) programs. The two programs gave very similar results (see Young et al., 1999 for evaluation of these and other programs concerned with phylogenetic-tree construction). Family assignments were based on the phylogenetic results and on the statistical analyses obtained with the GAP program (Devereux et al., 1984). The main features of the 10 families are presented in Table 1.

\section{RESULTS}

\section{The APC family: an overview}

Table 1 presents the 10 families of the APC superfamily. The names, abbreviations and TC numbers are provided for each family (columns 1 and 2). Column 3 tabulates the numbers of sequenced members of each family retrieved from the databases. Column 4 summarizes the source organisms from which the proteins of these families are derived. Column 5 presents the size ranges of the proteins in each family in numbers of aminoacyl residues per polypeptide chain. Column 6 lists the numbers of established or putative transmembrane $\alpha$ helical spanners (TMSs) in the proteins of these families, and column 7 provides, as an example, one functionally well-characterized member of each family, when available.

The families vary in size from 4 members [the EAT (ethanolamine transporter) family; TC 2.A.3.5] to 36 members [the AAT (amino acid transporter) family; TC 2.A.3.1]. Other relatively large families include the YAT (yeast amino acid transporter) family with 29 members, the LAT family with 26 members, the CAT family with 21 members and the APA (basic amino acid/polyamine transporter) family with 20 members. All remaining families are relatively small, the largest being the ACT (amino acid/choline transporter) family with 13 members. For an introduction to the TC system of transport protein classification recently approved by the transport nomenclature panel of the International Union of Biochemistry and Molecular Biology, see Saier (1999c, 2000b).

Of the 10 families, half of them are represented only 
Table 1. The 10 established families in the APC superfamily

\begin{tabular}{|c|c|c|c|c|c|c|}
\hline $\begin{array}{l}\text { Family } \\
\text { TC no. }\end{array}$ & Family name and abbreviation & $\begin{array}{c}\text { No. } \\
\text { members }\end{array}$ & $\begin{array}{c}\text { Source } \\
\text { organism* }\end{array}$ & $\begin{array}{c}\text { Size range } \\
\text { (no. residues) }\end{array}$ & No. TMSs $†$ & $\begin{array}{c}\text { Well- } \\
\text { characterized } \\
\text { example } \neq\end{array}$ \\
\hline 2.A.3.1 & Amino acid transporter, AAT & 35 & $\mathrm{~B}$ & $433-556$ & 12 & PheP Eco \\
\hline 2.A.3.2 & Basic amino acid/polyamine antiporter, APA & 20 & B & $418-501$ & 12 & PotE Eco \\
\hline 2.A.3.3 & Cationic amino acid transporter, CAT & 21 & $\mathrm{~B}, \mathrm{Pl}, \mathrm{An}$ & $462-657$ & $\begin{array}{l}14 \text { (eukaryotes); } \\
12 \text { (prokaryotes) }\end{array}$ & Cat1 Hsa \\
\hline 2.A.3.4 & Amino acid/choline transporter, ACT & 13 & $\mathrm{Y}, \mathrm{F}, \mathrm{Pl}$ & $517-618$ & 12 & GabA Eni \\
\hline 2.A.3.5 & Ethanolamine transporter, EAT & 4 & $\mathrm{~B}$ & $465-552$ & 12 & EntP Rer \\
\hline 2.A.3.6 & Archaeal/bacterial transporter, $\mathrm{ABT}$ & 8 & $\mathrm{~B}, \mathrm{Ar}$ & $424-736$ & 12 & - \\
\hline 2.A.3.7 & Glutamate: GABA antiporter, GGA & 9 & $\mathrm{~B}$ & $466-540$ & 12 & GadC Lla \\
\hline 2.A.3.8 & L-type amino acid transporter, LAT & 26 & An, Y & $440-662$ & 12 & Lat1 Rno \\
\hline 2.A.3.9 & Spore germination protein, SGP & 10 & Bacillus sp. & $358-382$ & 10 & GerXB Ban \\
\hline 2.A.3.10 & Yeast amino acid transporter, YAT & 29 & $\mathrm{Y}, \mathrm{F}$ & $521-852$ & 12 & Hip1 Sce \\
\hline
\end{tabular}

*B, bacteria; Ar, archaea; Y, yeast; F, fungi; An, animals; Pl, plants.

†Each family is believed to exhibit uniform topology except the CAT family, in which eukaryotic proteins have 14 TMSs while prokaryotic proteins have 12 .

$\ddagger$ See Table 3 for descriptions of these proteins.

Table 2. Interfamilial binary comparison scores

The GAP program with 100 random shuffles was used to generate the comparison scores reported, and these are expressed in standard deviations (SD). Percentage similarity values are also provided. The entire sequences of the proteins compared were used in these studies except for the comparisons presented below the dashed line, where scores were optimized by selection of the regions of highest similarity. These segments were always in excess of 100 residues in length.

\begin{tabular}{|c|c|c|c|c|c|c|}
\hline TC no.* & Protein 1 & TC no. & Protein 2 & $\begin{array}{c}\text { Comparison } \\
\text { score }(\mathrm{SD})\end{array}$ & $\begin{array}{l}\text { Identity } \\
(\%)\end{array}$ & $\begin{array}{c}\text { Similarity } \\
(\%)\end{array}$ \\
\hline 2.A.3.1 & AroP Eco & 2.A.3.10 & Can1 Sce & 50 & 38 & 51 \\
\hline 2.A.3.1 & AroP Eco & 2.A.3.6 & Orf1 Mth & 20 & 31 & 45 \\
\hline 2.A.3.1 & AroP Eco & 2.A.3.3 & AtrC1 Rpr & 14 & 27 & 40 \\
\hline 2.A.3.1 & AroP Eco & 2.A.3.2 & ArcD Lsa & 13 & 29 & 42 \\
\hline 2.A.3.3 & RocE Mtu & 2.A.3.8 & Lat1 Rno & 23 & 30 & 39 \\
\hline 2.A.3.3 & RocE Mtu & 2.A.3.5 & EutP Rer & 22 & 33 & 44 \\
\hline 2.A.3.2 & ArcD Lsa & 2.A.3.7 & XasA Eco & 9 & 29 & 40 \\
\hline 2.A.3.2 & ArcD Lsa & 2.A.3.9 & GerAB Bsu & 9 & 28 & 40 \\
\hline 2.A.3.5 & EutP Rer & 2.A.3.4 & Hnm1 Sce & 10 & 27 & 40 \\
\hline $\begin{array}{l}\text { 2.A.42.2.1 } \\
\text { (HAAAP family) }\end{array}$ & SdaC Eco & $\begin{array}{l}\text { 2.A.42.1.1 } \\
\text { (HAAAP family) }\end{array}$ & TyrP Ctr & 11 & 22 & 41 \\
\hline $\begin{array}{l}\text { 2.A.42.2.1 } \\
\text { (HAAAP family) }\end{array}$ & SdaC Eco & $\begin{array}{l}\text { 2.A.3.1.2 } \\
\text { (APC superfamily) }\end{array}$ & LysP Eco & 8 & 23 & 39 \\
\hline $\begin{array}{l}\text { 2.A.42.1.1 } \\
\text { (HAAAP family) }\end{array}$ & TyrP Ctr & $\begin{array}{l}\text { 2.A.3.9.2 } \\
\text { (APC superfamily) }\end{array}$ & GerBB Bsu & 7 & 22 & 39 \\
\hline $\begin{array}{l}\text { 2.A.42.1.2 } \\
\text { (HAAAP family) }\end{array}$ & Mtr Eco & $\begin{array}{l}\text { 2.A.18.4.1 } \\
\text { (AAAP superfamily) }\end{array}$ & Ynk1 Sce & 8 & 20 & 39 \\
\hline
\end{tabular}

* The TC numbers of the families within the APC superfamily are provided for all comparisons between proteins within the APC superfamily, presented above the dashed line. The individual proteins compared can be identified in Table 3 . The TC numbers of the proteins selected for the interfamilial comparisons reported below the dashed line are provided. Using our standard criteria, the values allow establishment of homology for the 10 APC families (above the dashed line) but not for the AAAP and HAAAP families (Saier, 1994). 
Table 3. Sequenced protein members of the 10 established families of the APC superfamily

\begin{tabular}{|c|c|c|c|c|c|}
\hline Protein & Description in database & Source organism & $\begin{array}{l}\text { No. } \\
\text { residues }\end{array}$ & $\begin{array}{c}\text { Accession } \\
\text { no.* }\end{array}$ & $\begin{array}{l}\text { NCBI gi } \\
\text { no. }\end{array}$ \\
\hline \multicolumn{6}{|c|}{ AAT family (TC 2.A.3.1) } \\
\hline Orf1 Asp & Putative transporter & Arthrobacter sp. & 466 & gbAB007122 & 3116225 \\
\hline YtnA Bsu & Amino acid transporter & B. subtilis & 463 & gbAF008220 & 2293166 \\
\hline AapA Bsu & Amino acid permease & B. subtilis & 459 & spO06005 & 3023220 \\
\hline YdgF Bsu & Hypothetical transporter & B. subtilis & 458 & spP96704 & 3025135 \\
\hline YbxG Bsu & Hypothetical transporter & B. subtilis & 462 & spP54425 & 3123299 \\
\hline GabP Bsu & $\gamma$-Aminobutyrate permease & B. subtilis & 469 & spP46349 & 2828523 \\
\hline HutM Bsu & Putative histidine permease & B. subtilis & 475 & spP42087 & 1170426 \\
\hline YbgF Bsu & Similar to histidine permease & B. subtilis & 470 & gbZ99105 & 2632526 \\
\hline RocE Bsu† & Amino acid permease & B. subtilis & 467 & spP39137 & 730601 \\
\hline RocC Bsu & Amino acid permease & B. subtilis & 470 & spP39636 & 730600 \\
\hline YvbW Bsu & Similar to amino acid permease & B. subtilis & 447 & spO32257 & 2635914 \\
\hline AroP Cgl & Aromatic amino acid transporter & $\begin{array}{l}\text { Corynebacterium } \\
\text { glutamicum }\end{array}$ & 463 & spQ46065 & 2492533 \\
\hline ProY Eco $†$ & Proline-specific permease & E. coli & 457 & spP77327 & 2492534 \\
\hline PheP Eco & Phenylalanine-specific permease & E. coli & 458 & spP24207 & 130068 \\
\hline CycA Eco† & D-serine/D-alanine/glycine transporter & E. coli & 470 & spP39312 & 732281 \\
\hline AroP Eco† & Aromatic amino acid transporter & E. coli & 457 & spP15993 & 2506093 \\
\hline YifK Eco & Probable transporter & E. coli & 461 & spP27837 & 2851572 \\
\hline AnsP Eco† & L-Asparagine permease & E. coli & 499 & spP77610 & 3183513 \\
\hline YkfD Eco & Hypothetical protein & E. coli & 475 & spQ47689 & 2492535 \\
\hline GabP Eco† & $\gamma$-Aminobutyrate permease & E. coli & 466 & spP25527 & 120778 \\
\hline LysP Eco† & Lysine-specific permease & E. coli & 489 & spP25737 & 547880 \\
\hline YeeF Eco & Hypothetical transporter & E. coli & 454 & spP33016 & 465570 \\
\hline YcjJ Eco & Hypothetical transporter & E. coli & 461 & spP76037 & 3915976 \\
\hline Orf1 Eco & Lysine-specific permease & E. coli & 433 & gbD90767 & 1742117 \\
\hline RocE Hpy & Amino acid permease & Hel. pylori & 519 & gbAE000610 & 2314162 \\
\hline Orf1 Hpy & Amino acid permease & Hel. pylori & 475 & gbAE001474 & 4154932 \\
\hline AnsP Mle & Putative L-asparagine permease & $\begin{array}{l}\text { Mycobacterium } \\
\text { leprae }\end{array}$ & 505 & gbAL035310 & 4200260 \\
\hline CycA Mtu & Putative transporter & Myc. tuberculosis & 556 & gbZ98268 & 2326747 \\
\hline AnsP Mtu & Putative transporter & Myc. tuberculosis & 489 & gbZ97559 & 2253066 \\
\hline Yao6 Mtu & Hypothetical protein & Myc. tuberculosis & 487 & spO06297 & 3024959 \\
\hline HutT Ppu & Inducible histidine transporter & Pseudomonas putida & 467 & gbAF032970 & 2642339 \\
\hline ProY Sty & Proline-specific permease & $\begin{array}{l}\text { Salmonella } \\
\text { typhimurium }\end{array}$ & 456 & spP37460 & 2506094 \\
\hline YifK Sty & Probable transporter & Sal. typhimurium & 461 & spP37456 & 586738 \\
\hline AnsP Sty & L-Asparagine permease & Sal. typhimurium & 497 & spP40812 & 728862 \\
\hline Orf48 Sty & Amino acid permease YeeF-like protein & Sal. typhimurium & 448 & gbX99945 & 1526981 \\
\hline Orf1 Sco & Putative L-asparagine permease & Streptomyces coelicolor & 489 & gbAL049587 & 4584481 \\
\hline \multicolumn{6}{|c|}{ APA family (TC 2.A.3.2) } \\
\hline Orf1 Asa & Polytopic cytoplasmic membrane protein & Aer. salmonicida & 426 & pirJC5086 & 2126237 \\
\hline ArcD Bli & Arginine: ornithine antiporter & B. licheniformis & 468 & gbY17554 & 3687418 \\
\hline YvsH Bsu & Amino acid permease & B. subtilis & 469 & gbZ99121 & 2635847 \\
\hline YkbA Bsu & Hypothetical transporter & B. subtilis & 438 & gbAJ002571 & 2632007 \\
\hline $\mathrm{ArcD} C \mathrm{Ctr}$ & Arginine: ornithine antiporter & Chl. trachomatis & 483 & gbAE001310 & 3328798 \\
\hline ArcD Cpn & Arginine: ornithine antiporter & $\begin{array}{l}\text { Chlamydia } \\
\text { pneumoniae }\end{array}$ & 485 & gbAE001683 & 4377363 \\
\hline ArcD Cpe & Arginine: ornithine antiporter & Clo. perfringens & 476 & spQ46170 & 3913092 \\
\hline LysI Cgl & L-Lysine transport protein & Cor. glutamicum & 501 & spP35865 & 547879 \\
\hline PotE Eco† & Putrescine: ornithine antiporter & E. coli & 439 & spP24170 & 130692 \\
\hline YjdE Eco & Hypothetical protein & E. coli & 445 & spP39269 & 2851627 \\
\hline CadB Eco† & Probable cadaverine: lysine antiporter & E. coli & 444 & spP23891 & 115415 \\
\hline ArcD Eco† & Arginine: ornithine antiporter & E. coli & 460 & spP77429 & 3913086 \\
\hline
\end{tabular}


Table 3 (cont.)

\begin{tabular}{|c|c|c|c|c|c|}
\hline Protein & Description in database & Source organism & $\begin{array}{l}\text { No. } \\
\text { residues }\end{array}$ & $\begin{array}{c}\text { Accession } \\
\text { no.* }\end{array}$ & $\begin{array}{l}\text { NCBI gi } \\
\text { no. }\end{array}$ \\
\hline YjeH Eco & Hypothetical transporter & E. coli & 418 & spP39277 & 731999 \\
\hline YhfM Eco & Hypothetical transporter & E. coli & 445 & spP45539 & 2851642 \\
\hline Orf1 Eco & Hypothetical transporter & E. coli & 462 & gbU18997 & 606304 \\
\hline PotE Hin & Putrescine: ornithine antiporter & Hae. influenzae & 435 & spP44768 & 1172565 \\
\hline ArcD Lsa & Arginine: ornithine antiporter & Lactobacillus sakei & 475 & spO53092 & 3913083 \\
\hline ArcD Pae† & Arginine: ornithine antiporter & $\begin{array}{l}\text { Pseudomonas } \\
\text { aeruginosa }\end{array}$ & 482 & spP18275 & 114116 \\
\hline PotE Rpr† & Putrescine: ornithine antiporter & Rickettsia prowazekii & 429 & gbAJ235272 & 3861036 \\
\hline OrfA Sco & Putative integral membrane transporter & Str. coelicolor & 474 & gbAL031515 & 3560003 \\
\hline \multicolumn{6}{|c|}{ CAT family (TC 2.A.3.3) } \\
\hline Aat1 Ath $\dagger$ & Amino acid transporter AAT1 & Ara. thaliana & 533 & pirS51171 & 1076291 \\
\hline Orf1 Ath & Putative amino acid transporter & Ara. thaliana & 569 & gbAC004238 & 3033391 \\
\hline YfnA Bsu† & YfnA protein & B. subtilis & 462 & gbD86418 & 2116759 \\
\hline YhdG Bsu & Hypothetical protein & B. subtilis & 465 & gbY14082 & 2226202 \\
\hline Orf7 Cel & Similar to amino acid permease & Cae. elegans & 589 & gbAF040642 & 2746784 \\
\hline Orf8 Cel & Similar to amino acid permease & Cae. elegans & 583 & gbAF024493 & 2394432 \\
\hline Orf9 Cel & Similar to amino acid permease & Cae. elegans & 585 & gbAF025452 & 2429392 \\
\hline CtrA Ddi & Similar to cationic amino acid transporter & $\begin{array}{l}\text { Dictyostelium } \\
\text { discoideum }\end{array}$ & 556 & gbAF015566 & 2353183 \\
\hline Cat1 Hsa† & Cationic amino acid transporter 1 & Homo sapiens & 629 & refNP_003036 & 4507047 \\
\hline Cat2 Hsa† & Cationic amino acid transporter 2 & Homo sapiens & 657 & refNP_003037 & 4507049 \\
\hline Cat4 Hsa & Cationic amino acid transporter 4 & Homo sapiens & 636 & refNP_004164 & 4759138 \\
\hline Err Mau & Ecotropic murine retrovirus receptor & Mesocricetus auratus & 628 & gbU26454 & 4096268 \\
\hline Cat1 Mmu & Cationic amino acid transporter & Mus musculus & 618 & gbU70859 & 1575776 \\
\hline Cat2 Mmu & Cationic amino acid transporter 2 & Mus musculus & 657 & pirA46073 & 1083250 \\
\hline RocE Mtu & RocE protein & Myc. tuberculosis & 476 & gbZ79702 & 1524280 \\
\hline Orf1 Mtu & Hypothetical protein & Myc. tuberculosis & 495 & gbZ95121 & 2072720 \\
\hline Cat1 Rno & Cationic amino acid transporter 1 & Rattus norvegicus & 624 & gbU70476 & 1589917 \\
\hline Cat2 Rnoł & Cationic amino acid transporter 2 (fragment) & Rat. norvegicus & 261 & gbU53927 & 1297194 \\
\hline Cat3 Rno & Cationic amino acid transporter 3 & Rat. norvegicus & 619 & gbAB000113 & 2116552 \\
\hline AtrC1 Rpr & Cationic amino acid transporter 1 & Ric. prowazekii & 470 & gbAJ23527 & 3860868 \\
\hline Orf1 Sco & Putative amino acid transporter & Str. coelicolor & 503 & gbAL023702 & 3169028 \\
\hline \multicolumn{6}{|c|}{ ACT family (TC 2.A.3.4) } \\
\hline Orf3 Ath & Putative amino acid or GABA permease & Ara. thaliana & 517 & gbAC006200 & 4262224 \\
\hline Gpt1 Cal† & Putative polyamine transporter & Candida albicans & 553 & gbAF080132 & 3415007 \\
\hline GabA Eni† & GABA permease & Emericella nidulans & 517 & gbAJ131668 & 4972245 \\
\hline Aap2 Ncr $†$ & Amino acid permease & Neurospora crassa & 551 & spO59942 & 3929337 \\
\hline Hnm1 Sce† & Choline transporter & Sac. cerevisiae & 563 & spP19807 & 117619 \\
\hline Bio5 Sce & $\begin{array}{l}\text { Transmembrane regulator of KAPA/DAPA } \\
\text { transporter }\end{array}$ & Sac. cerevisiae & 561 & spP53744 & 1730687 \\
\hline Uga4 Sce† & GABA-specific permease & Sac. cerevisiae & 571 & spP32837 & 418150 \\
\hline Ykr4 Sce & Hypothetical amino acid permease & Sac. cerevisiae & 618 & spP36029 & 549738 \\
\hline YahB Spo & Hypothetical amino acid permease & $\begin{array}{l}\text { Schizosaccharomyces } \\
\text { pombe }\end{array}$ & 544 & spQ09887 & 1351645 \\
\hline Yao8 Spo & Hypothetical amino acid permease & Schiz. pombe & 550 & spQ10087 & 1351705 \\
\hline Orf3 Spo & Putative amino acid permease & Schiz. pombe & 557 & gbAL031543 & 3581896 \\
\hline Orf4 Spo & Amino acid permease & Schiz. pombe & 554 & gbAL023595 & 3150116 \\
\hline Orf5 Spo & Amino acid permease & Schiz. pombe & 542 & gbAL023290 & 3116147 \\
\hline \multicolumn{6}{|c|}{ EAT family (TC 2.A.3.5) } \\
\hline Orf1 Mba† & Putative monomethylamine permease & Methanosarcina barkeri & 552 & gbAF013713 & 2746072 \\
\hline EutP Rer† & Ethanolamine permease & $\begin{array}{l}\text { Rhodococcus } \\
\text { erythropolis }\end{array}$ & 488 & gbL24492 & 403170 \\
\hline Orf1 Sme† & Hypothetical protein & Sinorhizobium meliloti & 465 & gbAF055582 & 3687686 \\
\hline EutP Zmo† & Ethanolamine permease & Zymomonas mobilis & 491 & gbAF124349 & 4378853 \\
\hline
\end{tabular}


Table 3. (cont.)

\begin{tabular}{|c|c|c|c|c|c|}
\hline Protein & Description in database & Source organism & $\begin{array}{l}\text { No. } \\
\text { residues }\end{array}$ & $\begin{array}{c}\text { Accession } \\
\text { no.* }\end{array}$ & $\begin{array}{l}\text { NCBI gi } \\
\text { no. }\end{array}$ \\
\hline \multicolumn{6}{|c|}{ ABT family (TC 2.A.3.6) } \\
\hline Cat1 Afu† & Putative cationic amino acid transporter 1 & Arc. fulgidus & 736 & gbAE000971 & 2648945 \\
\hline Cat2 Afu $\dagger$ & Putative cationic amino acid transporter 2 & Arc. fulgidus & 531 & gbAE000980 & 2648775 \\
\hline YbeC Bsu & Similar to amino acid transporter & B. subtilis & 539 & gbZ99105 & 2632498 \\
\hline YveA Bsu & Hypothetical protein & B. subtilis & 520 & gbZ94043 & 1945680 \\
\hline YbaT Eco† & Hypothetical protein & E. coli & 430 & spP77400 & 2495533 \\
\hline Y609 Mja† & Hypothetical protein & Met. jannaschii & 435 & spQ58026 & 2496073 \\
\hline Yw19 Mtu & Hypothetical protein & Myc. tuberculosis & 440 & spQ10858 & 1731248 \\
\hline Orf1 Mth & Hypothetical amino acid transporter & $\begin{array}{l}\text { Met. } \\
\text { thermoautotrophicum }\end{array}$ & 424 & gbAE000837 & 2621621 \\
\hline \multicolumn{6}{|c|}{ GGA family (TC 2.A.3.7) } \\
\hline XasA Cpn $†$ & Amino acid transporter & Chl.pneumoniae & 468 & gbAE001613 & 4376556 \\
\hline XasA Ctr & Amino acid transporter & Chl. trachomatis & 466 & gbAE001295 & 3328624 \\
\hline Orf54 Cpe & Membrane-spanning transport protein & Clo. perfringens & 495 & gbD49537 & 1402515 \\
\hline XasA Eco† & Amino acid antiporter & E. coli & 511 & spP39183 & 2507158 \\
\hline YjeM Eco & Hypothetical protein & E. coli & 514 & spP39282 & 732004 \\
\hline YgjI Eco & Hypothetical protein & E. coli & 477 & spP42590 & 1176115 \\
\hline Orf1 Eco & Putative transporter & E. coli & 540 & gbAE000192 & 1787127 \\
\hline GadC Lla & Putative glutamate: GABA antiporter & Lco. lactis & 503 & gbAF005098 & 2352486 \\
\hline XasS SflS & Amino acid antiporter & Shigella flexneri & 511 & spQ54152 & 2499135 \\
\hline \multicolumn{6}{|c|}{ LAT family (TC 2.A.3.8) } \\
\hline Orf1 Cel & Similarity to Schistosoma amino acid permease & Cae. elegans & 464 & gbU50308 & 1226279 \\
\hline Orf2 Cel & Similar to amino acid permeases & Cae. elegans & 563 & gbAF026214 & 2435610 \\
\hline Orf3 Cel & Similar to amino acid permeases & Cae. elegans & 566 & gbAF003144 & 2088757 \\
\hline Orf4 Cel & Similar to amino acid permeases & Cae. elegans & 562 & gbU56963 & 1293821 \\
\hline Orf5 Cel & Similarity to Schistosoma amino acid permease & Cae. elegans & 440 & $\mathrm{gbZ74042}$ & 3879689 \\
\hline Orf6 Cel & Similarity to amino acid permeases & Cae. elegans & 662 & gbAF040647 & 2746835 \\
\hline Orf7 Cel & Similarity to human membrane protein E16 & Cae. elegans & 493 & gbZ68216 & 3876345 \\
\hline Mnd Dme & Minidiscs/similar to amino acid transporter & $\begin{array}{l}\text { Drosophila } \\
\text { melanogaster }\end{array}$ & 499 & gbAF139834 & 5081597 \\
\hline $\mathrm{y}^{+}$Lat1 Hsa† & Unknown protein in locus pSLC7A6 & Homo sapiens & 515 & refNP 003974 & 4507053 \\
\hline $\mathrm{y}^{+}$Lat $2 \mathrm{Hsa}$ & Unknown protein in locus pSLC7A7 & Homo sapiens & 511 & refNP 003973 & 4507055 \\
\hline Orf1 Hsa & Membrane protein E16 & Homo sapiens & 507 & refNP 003477 & 4507051 \\
\hline Orf2 Hsa & $4 \mathrm{~F} 2$ light chain & Homo sapiens & 507 & gbAB017908 & 4519803 \\
\hline $\begin{array}{l}\text { hbO, }+ \text { AT } \\
\text { Hsa }\end{array}$ & $\begin{array}{l}\text { Putative glycoprotein-associated amino acid } \\
\text { transporter }\end{array}$ & Homo sapiens & 487 & NA $\|$ & $\mathrm{NA} \|$ \\
\hline hLAT2 Hsa† & LAT2 (L-type amino acid transporter 2) & Homo sapiens & 534 & $\mathrm{NA} \|$ & NA $\|$ \\
\hline hLAT3 Hsa & LAT3 putative amino acid transporter & Homo sapiens & 535 & gbY18483 & 4581470 \\
\hline $\mathrm{y}^{+}$Lat1 $\mathrm{Mmu}$ & Putative amino acid transporter & Mus musculus & 510 & gbAJ012754 & 3970791 \\
\hline Orf1 Mmu & 4F2/CD98 light chain & Mus musculus & 512 & gbAB017189 & 4519256 \\
\hline Xct Mmu & Cystine/glutamate transporter & Mus musculus & 502 & gbAB022345 & 4689081 \\
\hline $\begin{array}{l}\mathrm{mbO},+\mathrm{AT} \\
\mathrm{Mmu}\end{array}$ & $\begin{array}{l}\text { Putative glycoprotein-associated amino acid } \\
\text { transporter }\end{array}$ & Mus musculus & 487 & $\mathrm{NA} \|$ & $\mathrm{NA} \|$ \\
\hline $\begin{array}{l}\mathrm{mLAT2} \\
\mathrm{Mmu}\end{array}$ & LAT2 (L-type amino acid transporter 2) & Mus musculus & 531 & $\mathrm{NA} \|$ & NA $\|$ \\
\hline Lat1 Rno† & LAT1 (L-type amino acid transporter 1) & Rat. norvegicus & 512 & gbAB015432 & 3582136 \\
\hline Mup1 Sce† & High-affinity methionine permease & Sac. cerevisiae & 574 & spP50276 & 1709181 \\
\hline Mup3 Sce & $\begin{array}{l}\text { Hypothetical } 60 \cdot 6 \mathrm{kDa} \text { protein in CBP2-SSBR1 } \\
\text { intergenic region }\end{array}$ & Sac. cerevisiae & 546 & spP38734 & 731613 \\
\hline SPRM1 Sma† & Amino acid permease & Schistosoma mansoni & 503 & gbL25068 & 407047 \\
\hline ASUR4 Xla† & Amino acid transporter chain (AmAt-L-lc) ASUR4 & Xenopus laevis & 507 & gbY12716 & 3114983 \\
\hline Lat-1 Xla & L-Amino acid transporter 1 & $X$. laevis & 507 & gbAF019906 & 2731774 \\
\hline
\end{tabular}


APC superfamily

Table 3 (cont.)

\begin{tabular}{|c|c|c|c|c|c|}
\hline Protein & Description in database & Source organism & $\begin{array}{l}\text { No. } \\
\text { residues }\end{array}$ & $\begin{array}{c}\text { Accession } \\
\text { no.* }\end{array}$ & $\begin{array}{c}\text { NCBI gi } \\
\text { no. }\end{array}$ \\
\hline \multicolumn{6}{|c|}{ SGP family (TC 2.A.3.9) } \\
\hline GerXB Ban† & Spore germination protein GerXB & Bacillus anthracis & 359 & gbAF108144 & 4092083 \\
\hline GerIB Bsu† & Spore germination protein & Bacillus cereus & 364 & gbAF067645 & 3290176 \\
\hline YndE Bha & Spore germination protein (gene $728-1876$ ) & Bacillus halodurans & 382 & gbAB013370 & 4514335 \\
\hline GerAB Bmeg & Germination protein (gene 3086-4189) & Bacillus megaterium & 367 & gbU61380 & 2204286 \\
\hline GerAB Bsu† & Spore germination protein & B. subtilis & 365 & spP07869 & 1708033 \\
\hline GerBB Bsu & Spore germination protein & B. subtilis & 368 & spP39570 & 729628 \\
\hline Orf1 Bsu & Spore germination protein & B. subtilis & 363 & gbZ99113 & 2634160 \\
\hline GrkB Bsu† & Spore germination protein & B. subtilis & 373 & spP49940 & 1708044 \\
\hline YfkT Bsu & Putative germination protein & B. subtilis & 358 & gbD86417 & 2443222 \\
\hline \multicolumn{6}{|c|}{ YAT family (TC 2.A.3.10) } \\
\hline Aat1 Amu & Amino acid transporter & Amanita muscaria & 538 & gbAJ223504 & 4468097 \\
\hline Can1 Cal & Lysine/arginine permease & Can. albicans & 571 & spP43059 & 1168755 \\
\hline PutX Eni & Proline transport protein & Eme. nidulans & 570 & pirS42708 & 630383 \\
\hline Naap1 Ncr & Amino acid permease & N. crassa & 572 & gbAF001032 & 2197048 \\
\hline Lyp1 Sce† & Lysine-specific permease & Sac. cerevisiae & 611 & spP32487 & 1708894 \\
\hline Can1 Sce & Arginine permease & Sac. cerevisiae & 590 & spP04817 & 729014 \\
\hline Dip5 Sce & Dicarboxylic amino acid permease & Sac. cerevisiae & 608 & spP53388 & 1706439 \\
\hline Alp1 Sce & Basic amino acid permease & Sac. cerevisiae & 573 & spP38971 & 1703255 \\
\hline Gap1 Sce & $\gamma$-Aminobutyrate permease & Sac. cerevisiae & 601 & spP19145 & 544369 \\
\hline Tat2 Sce & Tryptophan permease & Sac. cerevisiae & 592 & spP38967 & 730893 \\
\hline Put4 Sce† & Proline-specific permease & Sac. cerevisiae & 627 & spP15380 & 1709941 \\
\hline Agp1 Sce & Asparagine/glutamine permease & Sac. cerevisiae & 633 & spP25376 & 140359 \\
\hline Hip1 Sce* & Histidine permease & Sac. cerevisiae & 603 & spP06775 & 729714 \\
\hline Bap3 Sce & Valine amino acid permease & Sac. cerevisiae & 604 & spP41815 & 1709572 \\
\hline Gnp1 Sce & High-affinity glutamine permease & Sac. cerevisiae & 663 & spP48813 & 1708002 \\
\hline Agp3 Sce† & General amino acid permease & Sac. cerevisiae & 558 & spP43548 & 1175959 \\
\hline Bap2 Sce & Leucine/valine/isoleucine amino acid permease & Sac. cerevisiae & 609 & spP38084 & 586506 \\
\hline Val1 Sce & Valine/tyrosine/tryptophan amino acid permease & Sac. cerevisiae & 619 & spP38085 & 586507 \\
\hline Mmp1 Sce & $S$-methylmethionine transporter & Sac. cerevisiae & 583 & pirS50959 & 1077318 \\
\hline Sam3 Sce & $S$-adenosylmethionine transporter & Sac. cerevisiae & 587 & pirS65307 & 2133015 \\
\hline Agp2 Sce & General amino acid permease & Sac. cerevisiae & 596 & spP38090 & 586542 \\
\hline Ssy1 Sce & Putative amino acid permease & Sac. cerevisiae & 852 & spQ03770 & 3024669 \\
\hline Isp5 Spo & Putative amino acid permease/sexual differentiation & Schiz. pombe & 543 & spP40901 & 729860 \\
\hline Orf2 Spo & Putative amino acid permease & Schiz. pombe & 521 & gbAL031532 & 3560241 \\
\hline Orf1 Spo & Amino acid permease & Schiz. pombe & 574 & gbAL023594 & 3150139 \\
\hline Aap1 Spo† & Amino acid permease & Schiz. pombe & 594 & gbD87954 & 1620894 \\
\hline Orf3 Spo & Amino acid permease & Schiz. pombe & 537 & gbAL023590 & 3136040 \\
\hline Ina1 Tha† & Amino acid permease & $\begin{array}{c}\text { Trichoderma } \\
\text { harzianum }\end{array}$ & 573 & spP34054 & 462414 \\
\hline Orf1 Ufa & Putative permease & Uromyces fabae & 561 & gbU81794 & 1764098 \\
\hline
\end{tabular}

*gb, GenBank; sp, SWISS-PROT.

† Included in the APC superfamily phylogenetic tree (Fig. 1).

$\ddagger$ Excluded from phylogenetic analysis because the sequence is incomplete.

\$Excluded from phylogenetic analysis due to $100 \%$ identity with XasA Eco (2507158).

\| Not available; sequence obtained from Dr F. Verrey (Verrey et al., 1999).

9 Sequencing errors in GenBank entry have been corrected. 


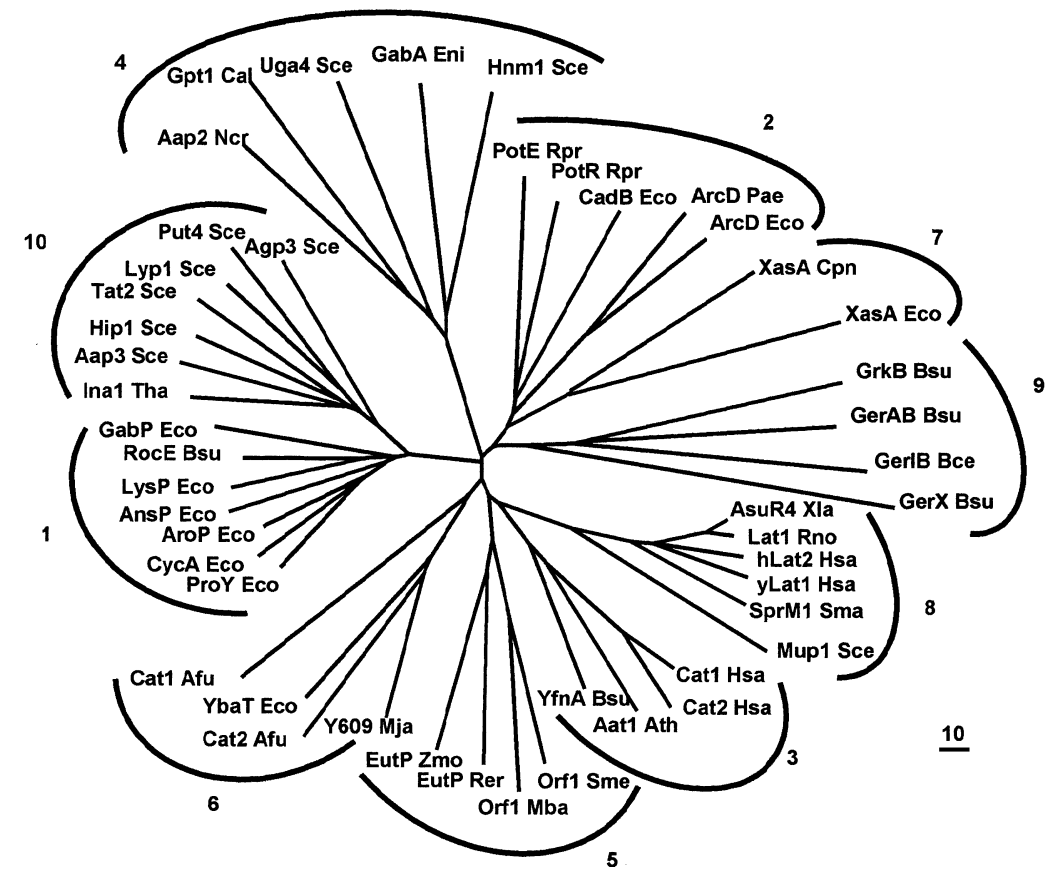

Fig. 1. Phylogenetic tree of the entire APC superfamily, showing segregation of the proteins into the 10 clusters that define the 10 constituent families. At least four representative proteins from each of the 10 families were included in the analysis with the sole exception of the GGA family, where only two proteins were included. The TREE program (Feng \& Doolittle, 1990) was used to generate this and other trees reported in this paper, as well as the multiple alignments upon which the hydropathy profiles (Fig. 2) and signature sequences (Table 4) were based. Groups: 1, AAT family; 2, APA family; 3, CAT family; 4, ACT family; 5, EAT family; 6, ABT family; 7, GGA family; 8, LAT family; 9, SGP family; 10, YAT family. The bar represents phylogenetic distance in arbitrary units and is the same relative length in all figures.

in bacteria. One family, the ABT (archaeal/bacterial transporter) family, is found only in bacteria and archaea but not eukaryotes. The CAT family is found in bacteria and eukaryotes but not archaea. Finally, three families are found only in eukaryotes (the ACT, LAT and YAT families). While YAT family members are derived exclusively from the fungal kingdom (yeast, fungi and mushrooms), ACT family members are derived from both plants and fungi but not animals, and LAT family proteins occur in both fungi and animals but not plants. Thus, all animal proteins are included in only two families, all plant proteins are similarly included in just two families, but fungal proteins are included in three families. Four of the five largest families are those which have representation in eukaryotes, but the largest family is specific to bacteria. The four smallest families are prokaryote specific.

Without exception, the five families specific to bacteria include members with the smallest polypeptide chains. The smallest of these proteins are restricted to the SGP family (size range 329-373 residues). No member of any other family is this small and this is the only family in which members have less than 12 putative TMSs. Proteins of the SGP family display just 10 TMSs. For the remaining four bacterial families, the protein size range is nearly the same (418-556 residues). Surprisingly, the ABT family shows a size range of 422-736 residues. Finally, the four families represented in eukaryotes show considerable size variation (440-852 residues). One of these families, the CAT family, exhibits the unique characteristic that all of the eukaryotic members display 14 putative TMSs although the prokaryotic members of this same family display the more usual 12 TMSs. Thus, eight families include members that exhibit 12 TMSs, one family consists of proteins with either 14 or 12 TMSs and one includes members with 10 TMSs. Size and topological variation will be analysed in greater detail below.

\section{Establishment of homology}

Table 2 summarizes the results of the statistical analyses of interfamilial binary sequence comparisons. As discussed previously (Saier, 1994, 1996), a comparison score of 9 standard deviations (SD) for a stretch of at least 60 residues in comparable regions of two proteins is considered sufficient to establish homology. The odds that this degree of similarity could have arisen by chance is 1 in $10^{-19}$. We and others have published the arguments leading to the conclusion that a value of $9 \mathrm{SD}$ could not have resulted either by chance or by a process of evolutionary sequence convergence (Doolittle, 1986; Saier, 1994). This conclusion is corroborated by the related functions and structures of the members of the APC superfamily. The results summarized in Table 2 therefore establish by these criteria that all 10 families listed in Table 1 include homologous proteins that are constituents of a single superfamily.

We have presented preliminary evidence that the eukaryote-specific amino acid/auxin permease (AAAP) family (TC 2.A.18) is distantly related to the APC family and that a small bacterial family, the hydroxy/aromatic amino acid permease (HAAAP) family (TC 2.A.42) is also a distant constituent of this superfamily (Young et al., 1999). In Table 2 we present statistical analyses that further support this suggestion.

The entries below the dashed line in Table 2 show that when SdaC of Escherichia coli was compared with TyrP of Chlamydia trachomatis, a comparison score of 11 SD was obtained. This value is sufficient to establish that these two proteins belong to a single family. Other 
comparisons reported in Table 2, where members of the HAAAP family are compared with members of the APC and AAAP families, do not reach a value of 9 SD. The comparison scores therefore do not allow us to establish homology between these families. The values of 7-8 SD obtained, however, are sufficient to strongly suggest homology. We therefore suggest that the AAAP and HAAAP families are distant constituents of the APC superfamily, although we cannot prove this contention statistically.

\section{Sequenced members of the APC superfamily}

Table 3 shows the identified, sequenced members of the 10 established families that comprise the APC superfamily. The table presents the abbreviations of the proteins to be used in this study, the database descriptions of these proteins, their source organisms, their sizes, and their database accession numbers as well as their GenBank identifier (gi) numbers. Analyses of the sequences of the listed proteins provide the basis for the conclusions presented in this paper.

\section{APC superfamily tree}

Fig. 1 presents a phylogenetic tree for the entire APC superfamily, where representative members of each family are included. Deep-rooted branching provides the phylogenetic basis for dividing the superfamily into its 10 constituent families. Thus, each family stems from a point near the centre of the unrooted tree. Further, all of the members of a family are more closely related to each other than any one of these members is related to a member of any other family. The family designations (see Table 1) were based on the available functional data as well as on the source organisms.

\section{Topological predictions}

Fig. 2 presents average hydropathy plots for all 10 families of the APC superfamily. These plots were based on multiple alignments generated for the full-length proteins of each family presented in Table 1 . Several features of these plots are worth mentioning. (1) The plots reveal that eight of the families display 12 putative TMSs with relative positions and relative sizes of the peaks being fairly similar. This fact shows that the sizes of both the TMSs and the inter-TMS loops have in general been conserved during evolution of the superfamily. (2) The CAT family (TC 2.A.3.3) displays 14 hydrophobic peaks due to the presence of two additional putative TMSs localized to the extreme $\mathrm{C}$ termini of the eukaryotic proteins of this family. This trait proved to be a characteristic only of the eukaryotic proteins, as all prokaryotic members of the CAT family exhibited the more usual 12 putative TMSs. No member of any other family within the APC superfamily exhibited this 14-TMS characteristic. (3) The SGP family (TC 2.A.3.9) displayed 10 putative TMSs rather than 12 . This proved to be due to a C-terminal truncation, resulting in the elimination of TMSs 11 and 12, present in all other members of the APC superfamily. The 10-TMS topology is therefore characteristic of the SGP family. (4) The hydropathy plots revealed that one member of the ABT family (TC 2.A.3.6; Cat1 Afu, see Table 3) exhibits a long C-terminal hydrophilic extension, while some members of the YAT family (TC 2.A.3.10) exhibit long $\mathrm{N}$-terminal hydrophilic extensions. The C-terminal extension of 200 aminoacyl residues in Cat1 Afu proved to be a conserved, duplicated, hydrophilic domain found in numerous archaeal, bacterial and eukaryotic proteins. Similarly, the N-terminal hydrophilic extensions of the yeast permeases of the YAT family proved to exhibit sequence similarity with conserved domains in other proteins. These hydrophilic N- and C-terminal domains undoubtedly serve important functions, possibly in promoting protein-protein interactions. Additionally, members of the CAT and GGA (glutamate:GABA transporter) families (TC 2.A.3.3 and 2.A.3.7, respectively) have short $\mathrm{N}$-terminal extensions, members of the EAT family (TC 2.A.3.5) have short C-terminal extensions and members of the AAT and ACT families (TC 2.A.3.1 and 2.A.3.4, respectively) have short N- and C-terminal extensions. Whether or not these hydrophilic extensions serve a specific function has yet to be examined. Members of remaining families (APA, TC 2.A.3.2; LAT, TC 2.A.3.8; and SGP, TC 2.A.3.9) lack or have only very short hydrophilic extensions at both ends of the proteins. (5) According to biochemically established topological features of representative prokaryotic APC superfamily members, both the $\mathrm{N}$ and $\mathrm{C}$ termini of the proteins are located in the cytoplasm with a 12 TMS topology (Cosgriff \& Pittard, 1997; Ellis et al., 1995; Hu \& King, 1998a). Loops connecting TMSs 1 and 2, 4 and 5, 9 and 10, and 11 and 12 are relatively short while remaining loops are longer. Three of these four short loops are putative extracytoplasmic loops while one of them is localized to the cytoplasm. The remaining loops appear to be of variable sizes and may be characteristic of the individual families. Nevertheless, extracytoplasmic loops are, on average, substantially shorter than cytoplasmic loops. In summary, both the degrees of relative hydrophobicity of the TMSs and the relative loop sizes are generally characteristic of the APC superfamily as a whole, with greatest constancy with respect to relative degrees of hydrophobicity of the various TMSs. This feature serves as a 'footprint' characteristic of the APC superfamily.

The complete multiple alignments of the proteins that comprise each of the 10 families of the APC superfamily, upon which the average hydropathy plots shown in Fig. 2 were based, can be found at http://www-biology. ucsd.edu/ msaier/transport/phylo.html. These alignments were generated with the TREE program (Feng \& Doolittle, 1990). Examination of these multiple alignments reveals that in general, well-conserved regions in all of these alignments can be found in which some residues are fully conserved and many more are largely conserved. It is clear that the program used to generate these alignments has aligned the sequences correctly. 


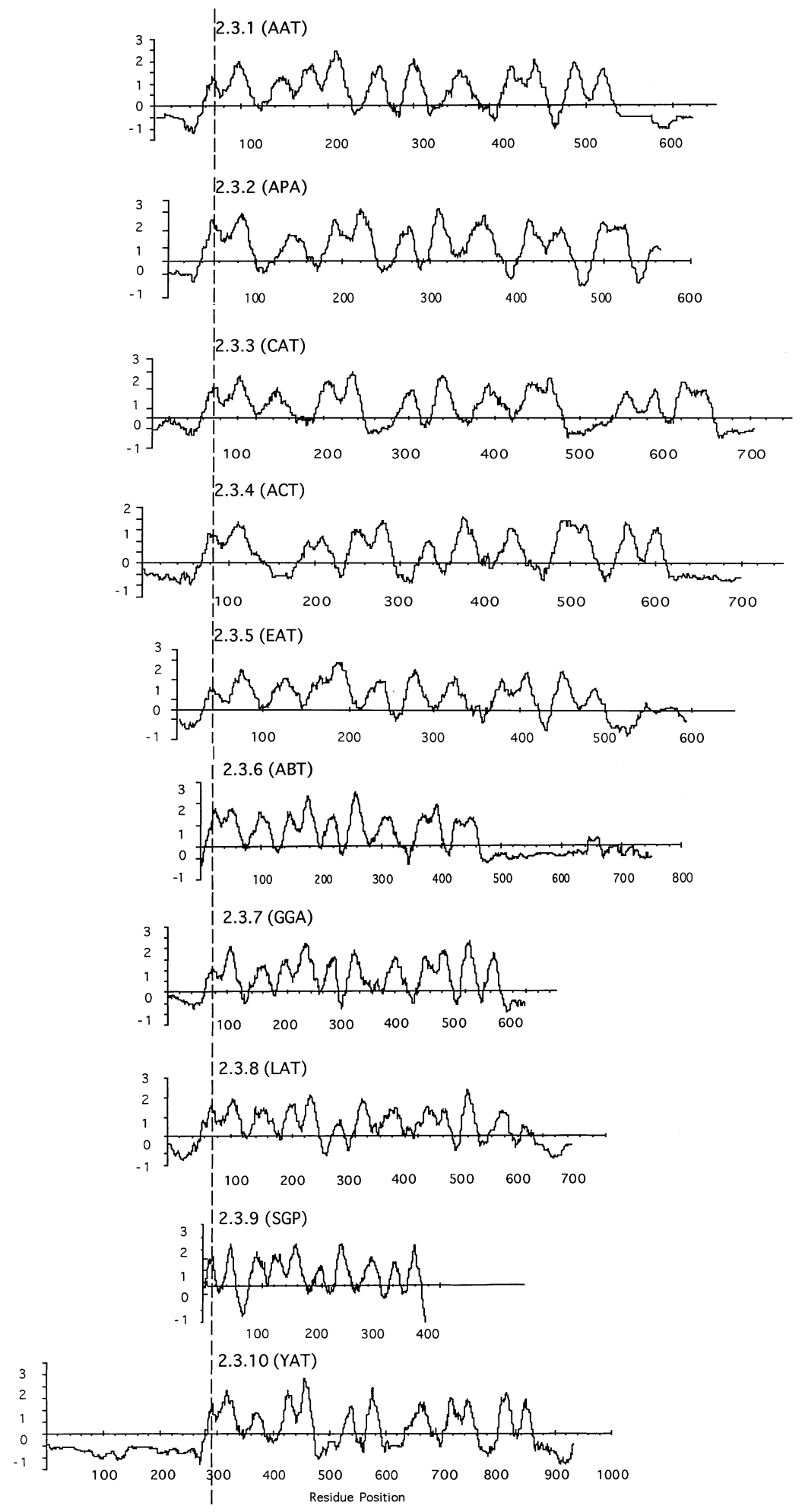

Fig. 2. Average hydropathy profiles for the proteins that comprise each of the 10 families of the APC superfamily. The program used was based on the hydropathy values for the amino acids reported by Kyte \& Doolittle (1982). A sliding window of 21 residues was used. In all of these plots, putative TMS no. 1 were aligned vertically to facilitate comparison of the results obtained for the 10 families. Individual proteins from all 10 families were examined using two additional hydropathy programs: TopPred and TMPred (Claros \& von Heijne, 1994; Hofmann \& Stoffel, 1993; von Heijne, 1992). The results confirmed the topological predictions shown here.

\section{APC superfamily signature sequences}

Table 4 presents signature sequences for the proteins of the 10 families within the APC superfamily. These sequences serve as identification motifs for these families. They were screened against the SWISS-PROT and TREMBL databases and retrieved only members of the represented family. By this criterion, they are bona fide signature sequences specific to these families. They can be used to identify additional members of these families as they are sequenced.

Several of the larger families in the APC superfamily are included within the Pfam (http://www.sanger.ac.uk/ Software/Pfam/) database of protein families as the 'amino acid permease' family (accession no. PF00324, 
Table 4. Signature sequences for the 10 families of the APC superfamily

\begin{tabular}{|c|c|c|}
\hline TC no. & Family & Signature sequence* \\
\hline 2.A.3.1 & AAT & $\begin{array}{l}\text { [PASDE]-X-[WH]-[LIVMY]-X(2)-[LIVA]-[LIVATSG]-X(2)-[LIVFGAT]-[LIVFA]-[LIVGMF]-X- } \\
\text { [LIVGAST]-X-N-[LIVFYMA]-X-[STANG]-[LIVT]-[KREQN]-X-[FYVT]-[GA]-[ENK]-X-[END] }\end{array}$ \\
\hline 2.A.3.2 & APA & $\begin{array}{l}\text { [FMVIL]-X(2)-[VCTSAG]-[LIVMA]-[LIVMSAN]-X-[TCLM]-[MFSVIL]-[FW]-[VCGAS]-[FY]-[TDVIL]- } \\
\text { G-[WMVIL]-[EAI]-[LBCAS]-[AFVIL] }\end{array}$ \\
\hline 2.A.3.3 & CAT & $\begin{array}{l}\text { [FMLIV]-[AS]-[GALIV]-[FLIVP]-[CS]-Y-[AGT]-E-[FLIV]-[GAS]-[SALIV]-[REAST]-[LIVF]-P-[LIVGKR]- } \\
\text { [AST]-G-[GS]-[ASLIV]-[FY] }\end{array}$ \\
\hline 2.A.3.4 & ACT & $\begin{array}{l}\text { [GPLST]-[WLIVFY]-[LIFV]-[LIVT]-[GASLIV]-[GACLIVM]-[ASPLIVF]-[LIVFCG]-X(2)-[LIVMFASC]- } \\
\text { [LIVTSC]-[GAS]-X-[GST]-[LIVM]-[GA]-[ED]-[LIVFH]-[LIVASC]-[AS]-X-[MFLIVWY]-P }\end{array}$ \\
\hline 2.A.3.5 & EAT & V-[LIV]-[SV]-G-[ED]-Y-[FAM]-G-W-[SN]-X-[STG]-[LIVW]-[GA]-X-[AG]-G \\
\hline 2.A.3.6 & $\mathrm{ABT}$ & [FSY]-[AST]-[KER]-[AL]-[ASG]-[SAL]-X-[LIVFMY]-[PV]-X(2)-[AG]-G-X-[LIVY]-X-[NFY]-X(5)-G \\
\hline 2.A.3.7 & GGA & G-[LIV]-X-[LIVAST]-W-[LIVATM]-[RSKN]-[DKNQSH]-[AST]-[LIVM]-G-X(2)-[WFLIV]-[GA]-[FY] \\
\hline 2.A.3.8 & LAT & $\begin{array}{l}\text { G-[WFY]-[DNFS]-X-[LIV]-[NH]-[FYT]-[LIVAGS]-[TALIV]-[EGPS]-E-[LIVM]-X[NDE]-P-X-[RK]-[NT]- } \\
\text { [LIVM]-[PK] }\end{array}$ \\
\hline 2.A.3.9 & SGP & $\begin{array}{l}\text { [FY]-[LIVF]-[ED]-[RH]-[LIVFS]-[ED]-[LYVFSI]-[LIVFM]-[LIVFMAS]-X-[LIVTFSAY]-X-[WLMY]- } \\
\text { [LIVAT]- [LIVMAP]-X(2)-[FYIM]-X(2)-[LIVFM]-[LIVAS]-[LIVMG] }\end{array}$ \\
\hline 2.A.3.10 & YAT & $\begin{array}{l}\text { E-X(2)-[FY]-[WACLIVFY]-[FLIVMAS]-[GASN]-X-[LIVMFCS]-K-[LIVA]-[LIVMGA]-[LIVSTAGF]- } \\
\text { [LIVMTA]-[LIVMTFCA]-[LIVMTGA]-[LIFYWM]-[LIFVTY]-[LIVF]-[LIVFTYC]-[LASGTCM]-[LIVF]- } \\
\text { [LIVC]-[LIVMTY]-X-[LIVFWCAS]-G }\end{array}$ \\
\hline
\end{tabular}

* Alternative residues at any one position are provided in square brackets. $\mathrm{X}$ indicates that any residue can occur at that position. Numbers in parentheses indicate numbers of positions. The one-letter abbreviations of the amino acids are used.

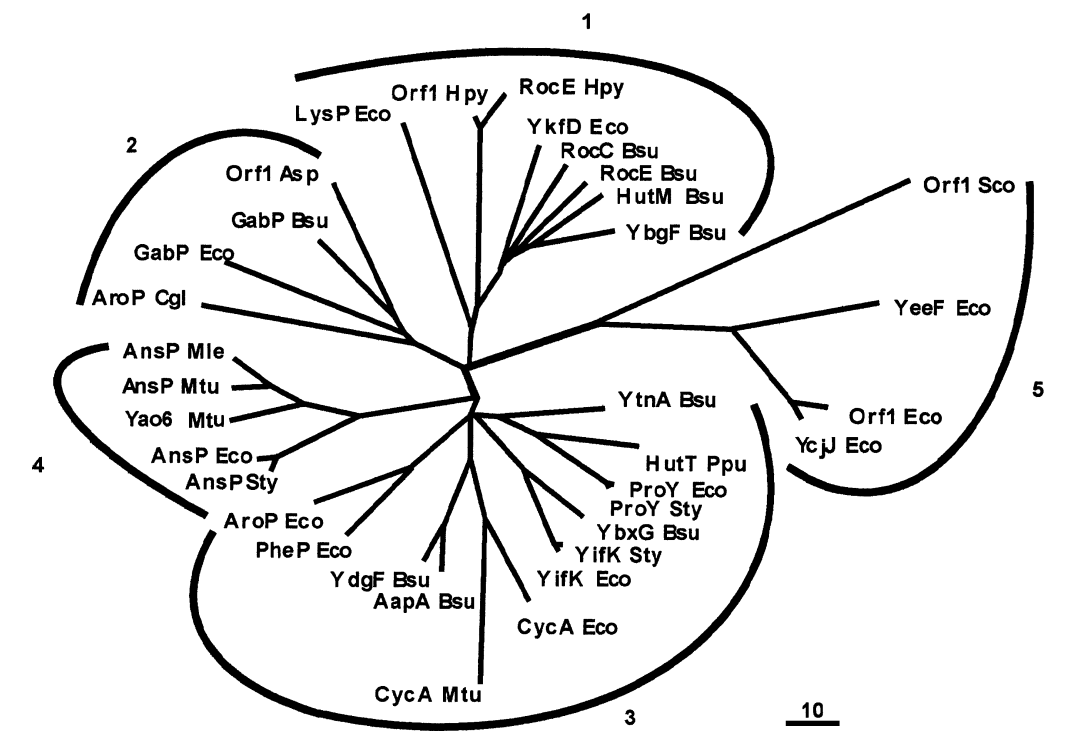

Fig. 3. Phylogenetic tree for the AAT (TC 2.A.3.1) family. prosite no. PDOC00191). However, the SGP family has been assigned to a distinct family (Pfam accession no. PB004126). Several of the smaller APC families are not included in the Pfam database (Bateman et al., 2000; Hofmann et al., 1999).

\section{Phylogeny of the 10 families within the APC superfamily}

Figs 3-12 present phylogenetic trees for the proteins of the 10 families within the APC superfamily. Evaluation of these trees provides evidence regarding paralogous and orthologous relationships. Thus, if divergence has arisen solely due to speciation, the genes are orthologues, but if they arose as a result of gene duplication events within a single organism, they are paralogues. The expected relative distances for orthologues can be estimated based on relative phylogenetic distances observed for the $16 \mathrm{~S}$ rRNAs from the organisms under consideration. It should be noted, however, that while most homologues in a single species can be assumed to be paralogues, it is more problematic to assign orthologous relationships for proteins from different species unless functional data are available. The reader should 


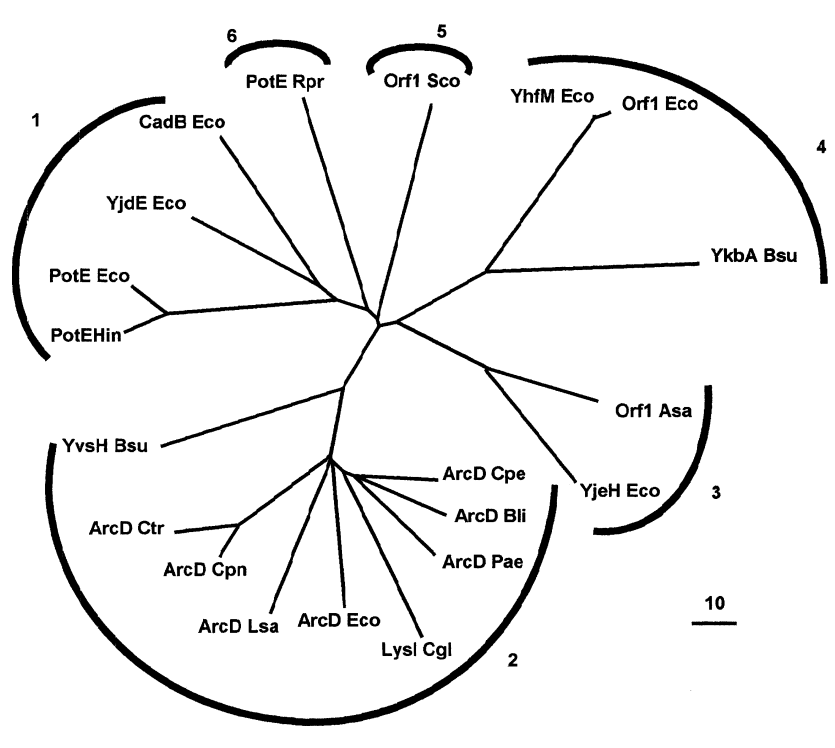

Fig. 4. Phylogenetic tree for the APA (TC 2.A.3.2) family.

therefore be cognizant of this problem when considering conclusions based on the phylogenetic trees presented below.

In Fig. 3 (the bacterial AAT family), there are five principal branches, and E. coli paralogues, of which there are 12, are found in all of these clusters. This fact suggests that gene duplication events occurred during the early evolution of this family. The nine Bacillus subtilis paralogues are found in three clusters and the three Mycobacterium tuberculosis paralogues appear in two of them. A few late gene-duplication events evidently gave rise to paralogues of very similar sequence. For example, PheP and AroP in cluster 3, the phenylalanine and aromatic amino acid permeases of E. coli, respectively, appear to have arisen by a gene-duplication event that probably occurred somewhat before Grampositive bacteria diverged from Gram-negative bacteria (judging from the distances observed for putative E. coli and B. subtilis orthologues). The four B. subtilis paralogues in cluster 1 probably arose after or at about the same time Gram-positive bacteria diverged from Gram-negative bacteria. The gene-duplication events giving rise to RocE and Orf1 of Helicobacter pylori (cluster 1), and YdgF and AapA of B. subtilis (cluster 3) must have occurred much more recently. Likely candidates for E. coli-B. subtilis orthologous pairs include YkfD Eco and RocC Bsu and its three close paralogues (cluster 1), GabP Eco and GabP Bsu (cluster 2), ProY Eco and YtnA Bsu, YifK Eco and YbxG Bsu, and CycA Eco and either AapA Bsu or YdgF Bsu (all in cluster 3). Only in the case of the two GabP orthologues (cluster 2) are functional data available to substantiate the conclusion of common function (Brechtel et al., 1996; Brechtel \& King, 1998; King et al., 1995).

Fig. 4 shows the tree for the bacterial APA family. Four clusters (1-4) and two long branches (5 and 6) can be seen. The seven E. coli paralogues appear in all four

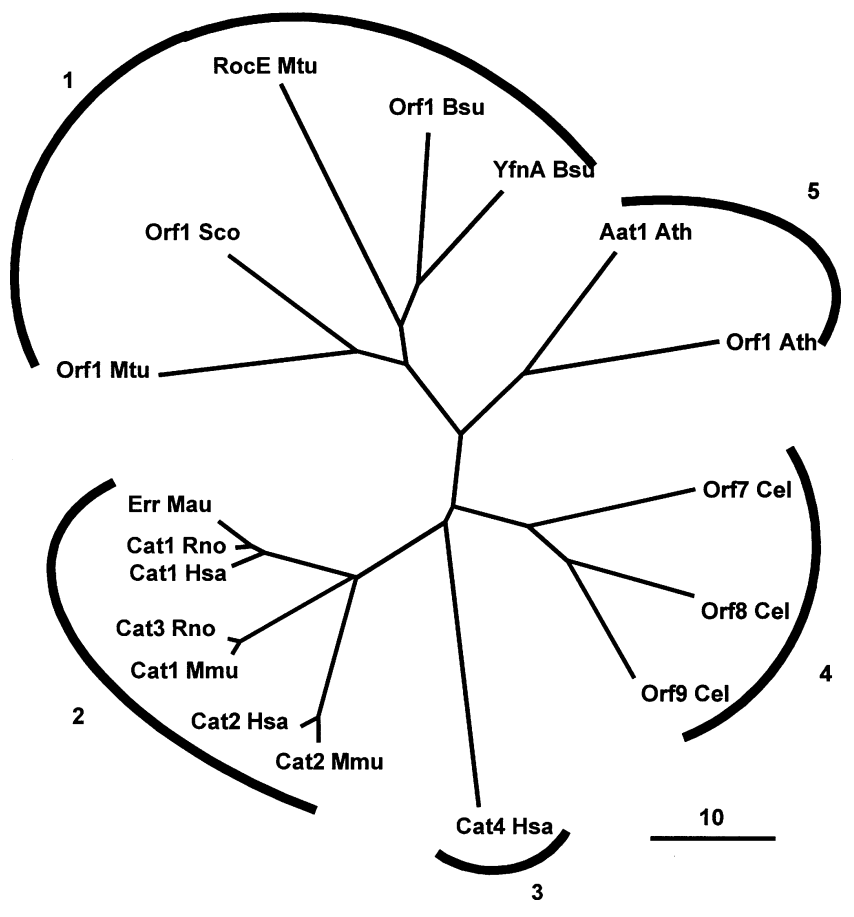

Fig. 5. Phylogenetic tree for the CAT (TC 2.A.3.3) family.

primary clusters. Thus, three E. coli paralogues (PotE, YjdE and $\mathrm{CadB}$ ) as well as a Haemophilus influenzae orthologue (PotE Hin), similar to PotE Eco, are found in cluster 1 . In the large cluster 2, no two members are from the same organism. Thus, no paralogous pairs are found in this cluster. Nevertheless, these proteins are clearly not all orthologues. For example, the great distance between Yvs Bsu and ArcD Bli, derived from B. subtilis and Bacillus licheniformis, respectively, suggests that these two proteins diverged following an early geneduplication event rather than as a result of speciation. Similarly, although seven of the nine proteins in cluster 2 have been annotated as ArcD proteins, the relative distances of these proteins do not generally correlate with the phylogenetic distances of the organisms. This fact suggests that they are not all orthologues of similar function. Cluster 3 consists of a probable pair of orthologues from E. coli and Aeromonas salmonicida, and cluster 4 consists of a possible orthologous pair from E. coli and B. subtilis (YkbA Bsu with either YhfM or Orf1 of E. coli). The two E. coli proteins in this cluster probably arose from a very recent gene-duplication event. Orf1 Sco (5) and PotE Rpr (6) are found at the ends of long branches. The great distance separating PotE Eco from PotE Rpr suggests that these two proteins are not orthologues. PotE Rpr may have been incorrectly annotated.

The tree shown in Fig. 5 for the CAT family shows clustering in accordance with the source organism. Thus, cluster 1 is specific for Gram-positive bacteria, clusters 2 and 3 include only mammalian members, cluster 4 is a group of three paralogues from the nematode Caenorhabditis elegans and cluster 5 includes 


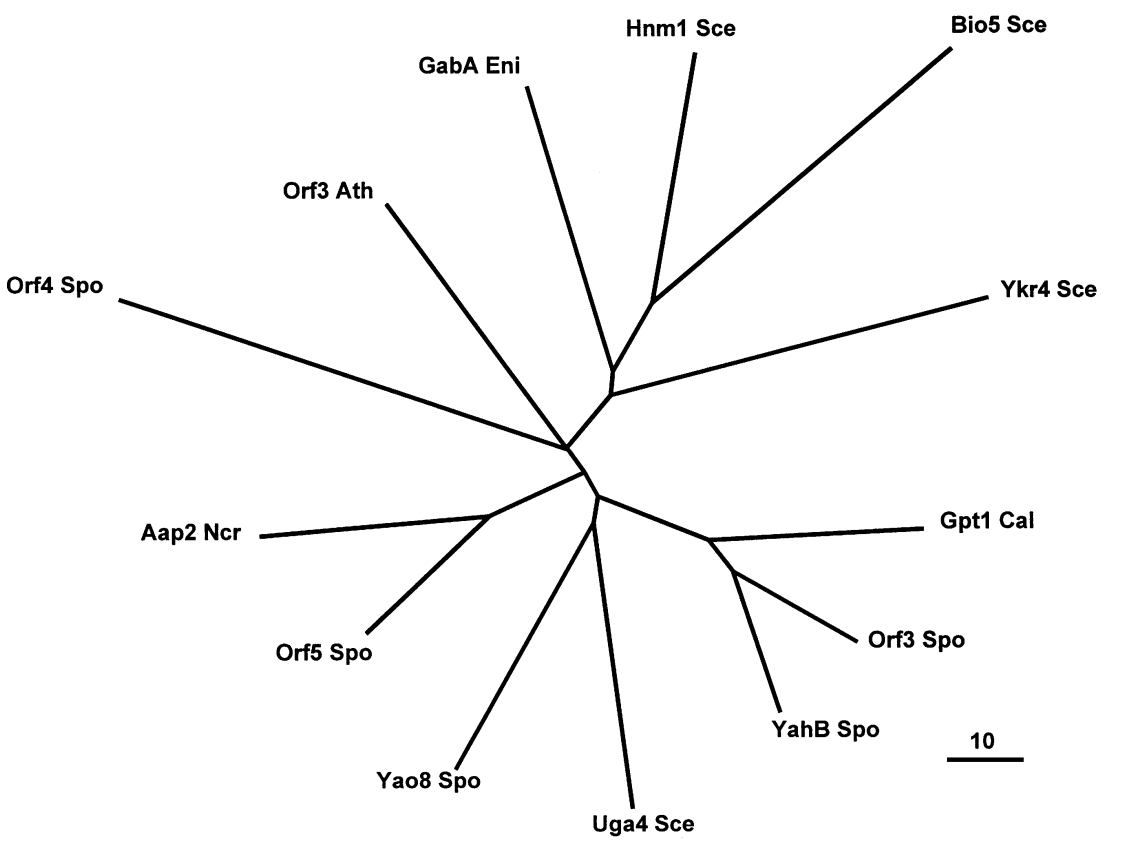

Fig. 6. Phylogenetic tree for the ACT (TC 2.A.3.4) family.

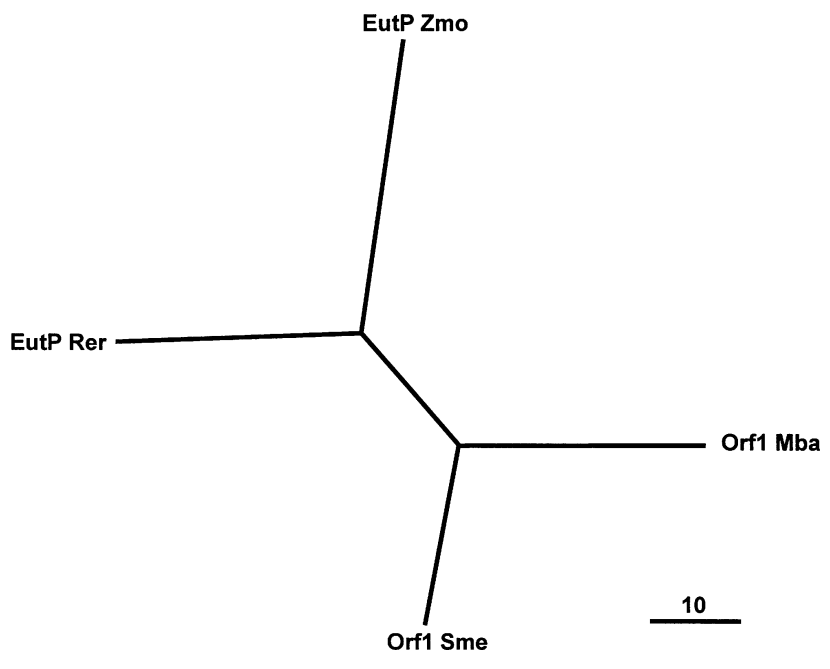

Fig. 7. Phylogenetic tree for the EAT (TC 2.A.3.5) family.

two paralogues from the plant Arabidopsis thaliana. Fig. 6 (the ACT family) shows a group of yeast and fungal proteins related to one plant protein. Little clustering is observed except that Aap2 Ncr and Orf5 Spo are likely to be orthologues, and the clustering of YahB Spo, Orf3 Spo and Gpt1 Cal suggests similar functions for these proteins. The EAT family tree (Fig. 7) is consistent with orthologous relationships between EntP Rer and EutP Zmo as well as between Orf1 Sme and Orf1 Mba.

The ABT family tree (Fig. 8) resembles the EAT family tree in that little clustering is observed. Neither the archaeal nor the bacterial proteins cluster together, suggesting that early gene duplication occurred before divergence of these two domains of prokaryotes. Alternatively, one could invoke lateral gene transfer to

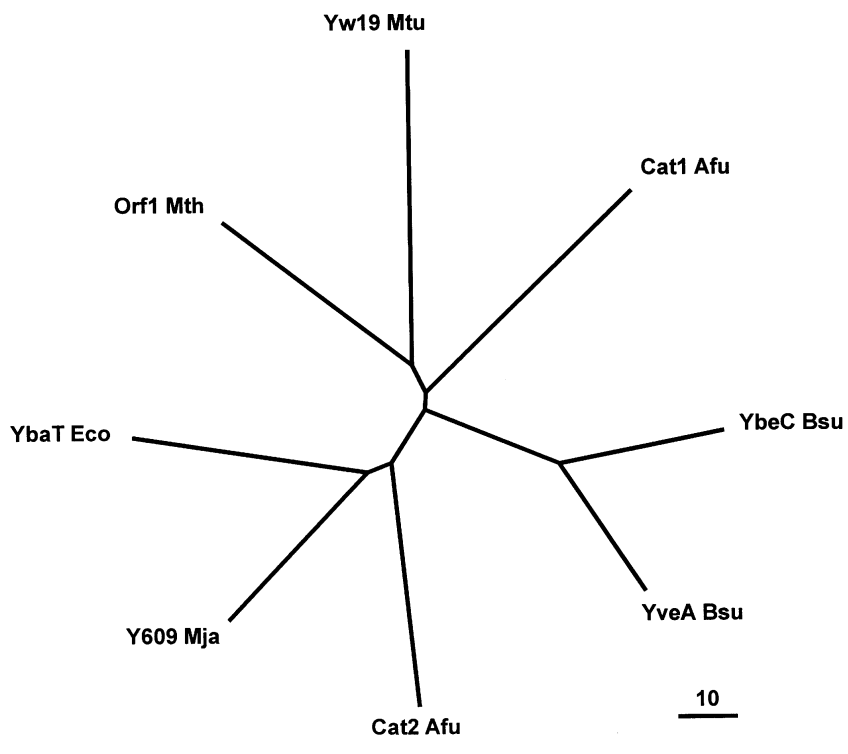

Fig. 8. Phylogenetic tree for the ABT (TC 2.A.3.6) family.

account for the results. The two B. subtilis paralogues probably arose by a relatively recent gene-duplication event.

In the GGA family tree (Fig. 9), the four E. coli paralogues are each found on distant branches. The XasA proteins of Chlamydia pneumoniae and Chl. trachomatis are undoubtedly orthologues, but the close clustering of XasA Eco and GadC Lla is surprising. Because E. coli and Lactococcus lactis are Gramnegative and Gram-positive bacteria, respectively, such close clustering would not be anticipated. We considered that this protein pair might provide an example of a recent lateral gene-transfer event between these two bacterial kingdoms. The same seemed possible for Orf54 


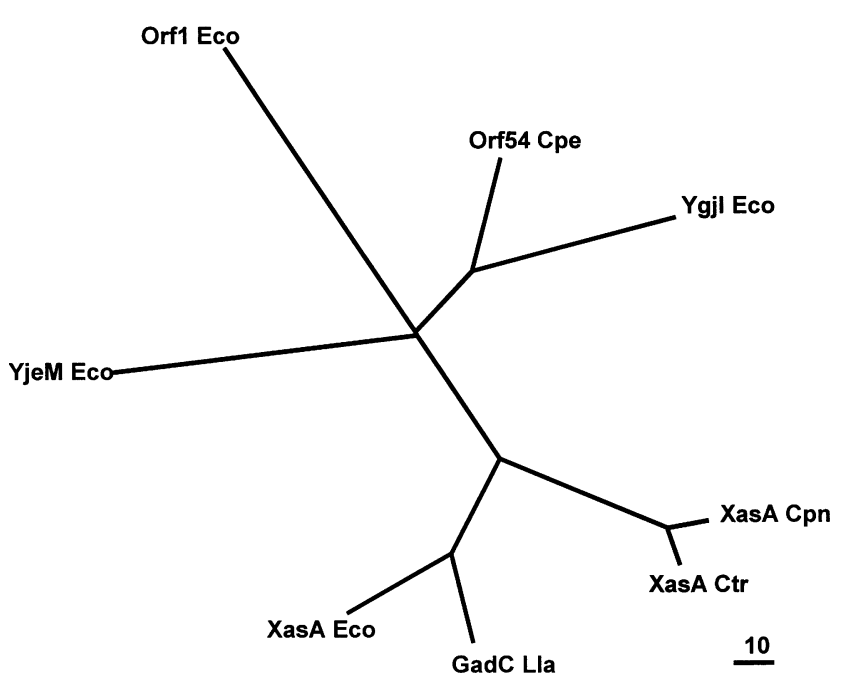

Fig. 9. Phylogenetic tree for the GGA (TC 2.A.3.7) family.

Cpe and YgjI Eco which, however, are more distant from each other. Indeed, examination of the $\mathrm{G}+\mathrm{C}$ contents of these genes revealed that the Gram-positive bacterial homologues exhibit the $\mathrm{G}+\mathrm{C}$ values expected for the source organisms $35.0 \mathrm{~mol} \%$ for the gadC gene of L. lactis compared to $35.4 \mathrm{~mol} \%$ expected for L. lactis genes, and $30.8 \mathrm{~mol} \%$ for the orf 54 gene of Clostridium perfringens compared to $31 \cdot 0 \mathrm{~mol} \%$ expected for Clo. perfringens genes), but the E. coli genes deviated significantly from expectation based on the $\mathrm{G}+\mathrm{C}$ content for E. coli genes as a whole $(46.0 \mathrm{~mol} \%$ for the $x a s A$ gene and $45.5 \mathrm{~mol} \%$ for the $y g j I$ gene compared to $51.4 \mathrm{~mol} \%$ expected for E. coli genes). In fact, the G + C contents of the two E. coli genes are nearly the same at each of the three nucleotide positions of the codons in the E. coli xasA and ygjI genes, and they differed considerably from those expected for native E. coli genes, suggesting that they were obtained horizontally from the same organism or two very similar organisms.

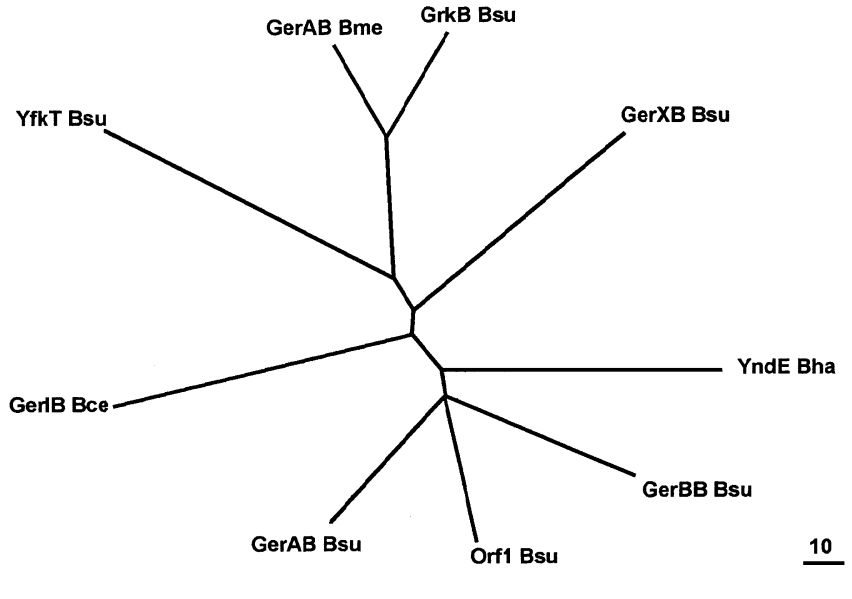

Fig. 11. Phylogenetic tree for the SGP (TC 2.A.3.9) family.

Codon usages for these two genes proved also to be more similar to each other than to E. coli genes (data not shown). These results suggest that E. coli acquired both of these genes from a Gram-positive bacterium long after Gram-positive and Gram-negative bacteria diverged from each other.

The phylogenetic tree for the large LAT family (Fig. 10), which includes animal and yeast proteins, reveals clustering patterns that in many cases reflect the phylogenies of the source organisms. Thus, the two yeast proteins, Mup1 and Mup3, cluster loosely together as do four ORFs from Cae. elegans. However, Cae. elegans proteins are found on two additional branches that cluster very loosely with the mammalian proteins. A number of mammalian orthologous and close paralogous relationships can be proposed on the basis of the tree configuration (see Fig. 10).

The bacterial SGP tree (Fig. 11) reveals some early and late gene-duplication events as well as one probable orthologous relationship for the various established and putative germination proteins. Thus, GrkB Bsu and

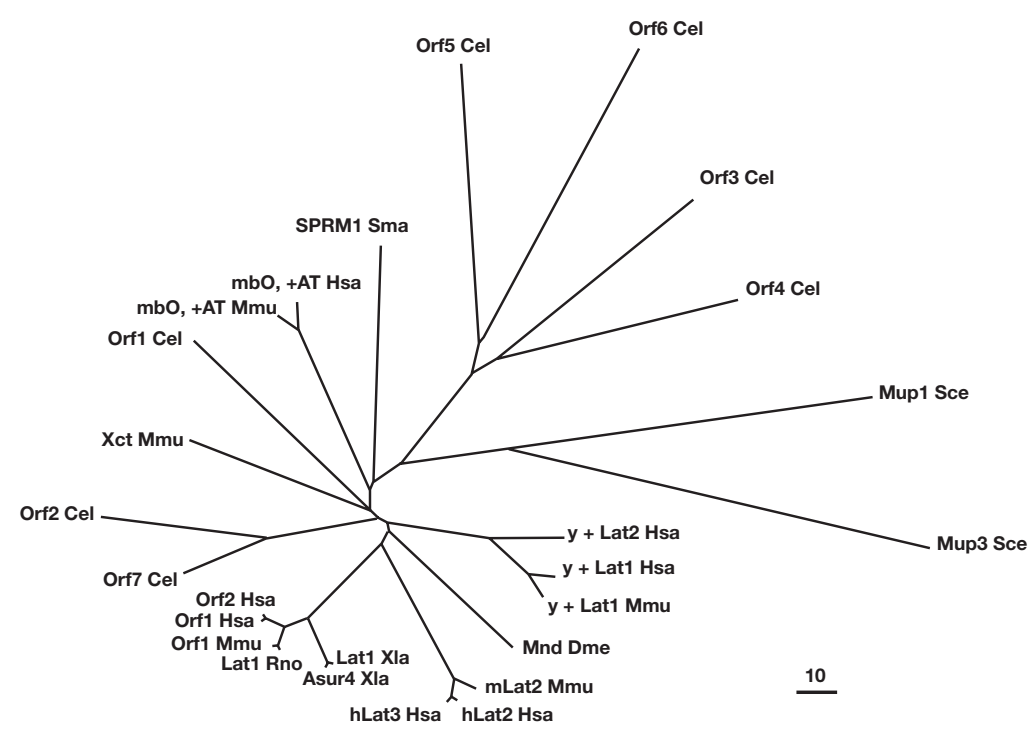

Fig. 10. Phylogenetic tree for the LAT (TC 2.A.3.8) family. 


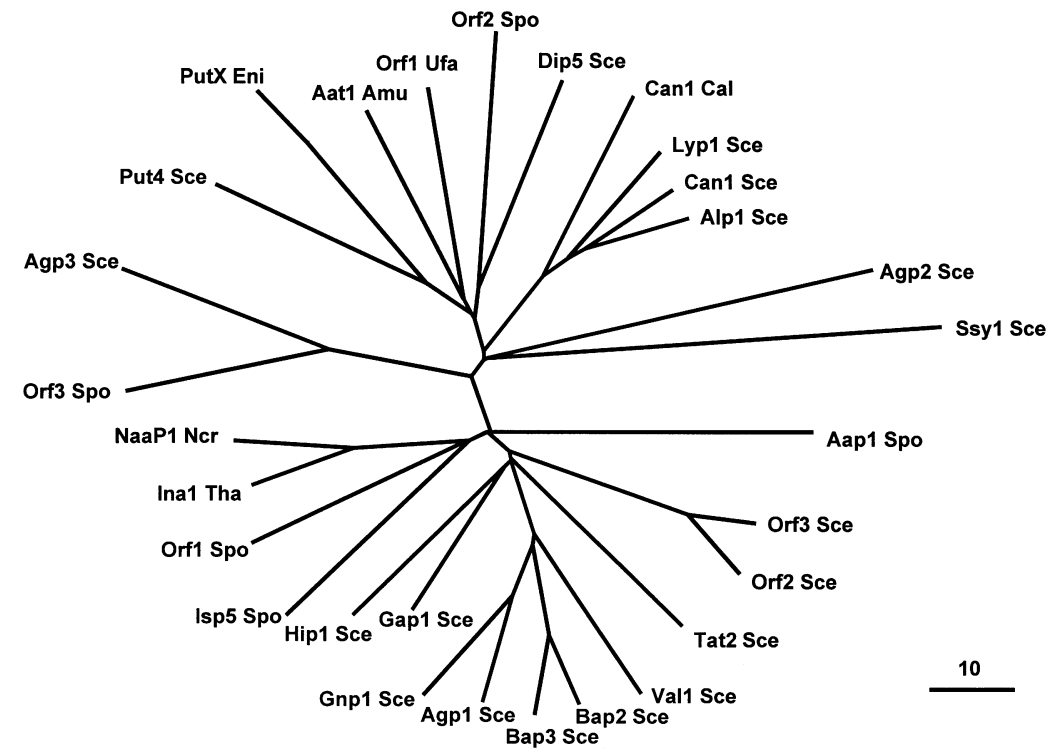

Fig. 12. Phylogenetic tree for the YAT (TC 2.A.3.10) family.
GerAB Bme are probable orthologues, but the cluster of three $B$. subtilis proteins at the bottom of the tree (GerAB, Orf1 and GerBB) represent paralogues that probably arose by recent gene-duplication events (Corfe et al., 1994; Zuberi et al., 1987). On the other hand, YfkT, GrkB and GerXB represent B. subtilis proteins that presumably arose by early gene-duplication events, maybe during the early origin of sporulation in the Bacillus line. This tree illustrates the tremendous amount of gene duplication that must have occurred in response to pressures arising during development of a program of prokaryotic differentiation. All of these proteins presumably arose by gene-duplication events in order to render spore germination responsive to the presence of amino acids in the growth medium.

Finally the YAT family (Fig. 12), where the vast majority of the proteins are from one species (Sac. cerevisiae), reveals a number of interesting orthologous and paralogous relationships. Particularly worthy of note is the huge cluster of 10 Sac. cerevisiae paralogues in a single cluster at the bottom of the tree. The occurrence of additional Sac. cerevisiae paralogues scattered throughout the tree is also noteworthy. Many of these yeast proteins are functionally characterized and they exhibit differing specificities. Thus, like bacilli, yeast has proliferated paralogues within the APC superfamily. Although many of these proteins are known to be active in the vegetative yeast cell, it will be interesting to determine if any of them function in the regulation of spore germination as has been demonstrated for the SGP family in Bacillus.

\section{DISCUSSION}

In this report we provide a detailed and up-to-date phylogenetic characterization of the APC superfamily. Based exclusively on phylogeny (Fig. 1), we identified 10 distinct families, five families specific to bacteria, one bacterial and archaeal family, one bacterial and eukaryotic family with representation only in animals and plants of the eukaryotic domain, and three eukaryote-specific families. Of the three eukaryotespecific families, one is fungi specific (including yeast and mushroom proteins), one includes members from both fungi and plants but not animals, and one includes members from both fungi and animals but not plants. Summarizing these observations, bacterial APC superfamily members are found in 7 of the 10 families, archaeal members are found in only one family, fungal proteins are found in three families, and both plant and animal members are each found in just two families.

\section{Protein size and topological comparisons}

Size and topological analyses showed that the SGP family (TC 2.A.3.9) includes the smallest proteins in the APC superfamily and these proteins display only 10 putative TMSs instead of the usual 12. The deficiency is at the $\mathrm{C}$ termini of these proteins. Interestingly, no member of this family has been shown to be a transporter, and the possibility that they serve as receptors rather than transporters can be entertained. Thus, loss of TMSs 11 and 12 may result in alteration of function. If, however, a transport function is demonstrated for one of these proteins, one can suggest that TMSs 11 and 12 in other APC superfamily permeases are not essential for transport.

One family, the CAT family, the only family to have representation in both prokaryotes and eukaryotes, shows a 12 TMS topology for the prokaryotic members but a 14 TMS topology for the eukaryotic members. Why these eukaryotic proteins, but no other members of the APC superfamily, have two extra putative TMSs is not known. Because the bacterial 12 TMS proteins 
cluster separately from the eukaryotic proteins on the CAT family tree (Fig. 5), it is reasonable to propose that the addition of two extra TMSs to the $\mathrm{C}$ termini of these proteins occurred only once during the evolution of the APC superfamily. It is possible that this C-terminal extension is related to the viral-receptor function of several of these proteins, although it could not have evolved to serve this function (see Reizer et al., 1993).

\section{Functionally uncharacterized families}

No functional data are available for members of one of the APC superfamily families, the ABT family (TC 2.A.3.6). This family is represented in three archaea (Methanococcus jannaschii, Methanobacterium thermoautotrophicum and Archaeoglobus fulgidus) and three bacteria (E. coli, B. subtilis and Myc.tuberculosis). We can assume that these proteins transport amino acids and/or their derivatives, but direct experimentation will be necessary to establish this assumption. Similarly, although proteins of the SGP family (TC 2.A.3.9) have clearly been implicated in spore germination, there is no evidence as to whether these proteins function in transport or as transmembrane receptors, signalling the presence of amino acids to cytoplasmic regulatory proteins. Determination of the biochemical functions of these proteins will prove of interest for a more complete understanding of the mechanisms regulating endospore germination in $\mathrm{Ba}$ cillus species.

\section{Distant phylogenetic relationships}

Although we were able to establish homology for all of the members of the APC superfamily included in this study, we also extended an earlier suggestion (Young et al., 1999) that two other recognized amino acid transporter families are distantly related to the APC superfamily (Table 2; Saier, 2000a). Thus, the eukaryotic AAAP family and the prokaryotic HAAAP family are most likely distant constituents of the APC superfamily although maximal comparison scores of only 7-8 SD were obtained when interfamilial comparisons with any established member of the APC superfamily were done. The PSI-BLAST program with iterations (Altschul et al., 1997), which uses a matrix/motif algorithm to detect distant phylogenetic relationships, also revealed similarities between members of these three families (unpublished results). It is therefore highly likely that the APC superfamily includes at least 12 recognized families instead of just 10, and because the AAAP family tree is complex, with several major sequence-divergent phylogenetic clusters (Young et al., 1999), the AAAP family may appropriately be subdivided into more than one subfamily. However, because we prefer to adhere to a rigorous standard for establishment of homology, we will retain the AAAP and HAAAP families in the TC system separate from the APC superfamily. No evidence for a phylogenetic relationship between the APC superfamily and the many other families of transporters capable of transporting amino acids and their derivatives has been forthcoming (Saier, 2000a).

It is interesting to note that members of the AAAP and HAAAP families exhibit 11 established or putative TMSs (see Young et al., 1999 for a summary of the biochemical and computational evidence). This number is different from that observed for any one of the established members of the APC superfamily. This fact suggests that the former two families may be more closely related to each other than to established members of the APC superfamily. Assuming all of these families to be related, we conclude that members of the extended APC superfamily may exhibit $10,11,12$ or 14 TMSs. The functional implications of this finding have yet to be elucidated.

\section{Concluding remarks}

Although the reported studies have revealed that the APC superfamily is one of the largest superfamilies found in nature, it has apparently not diversified greatly in substrate specificity. All of its functionally characterized members transport amino acids and/or their derivatives. In view of the broad specificities of permeases in certain other superfamilies such as the larger major facilitator superfamily (TC 2.A.1) (Pao et al., 1998; Saier et al., 1999a) or the smaller solute: sodium symporter family (TC 2.A.21), it is surprising that the APC superfamily has not diverged more extensively with respect to substrate recognition. An explanation for this observation is not currently at hand, but protein architectural constraints could be responsible (see Saier 1994, 1996, 1998).

Future DNA sequencing projects and functional analyses of the revealed genes will undoubtedly result in both numerical and functional expansion of the 10 currently established families of the APC superfamily. Moreover, we can expect that completely novel families within the APC superfamily will be discovered. Evolutionary links to other families such as the AAAP family may also become unequivocally established as 'missing-link' sequences become available. Additionally, more refined in silico tools for establishing homology as well as high resolution three-dimensional structural data for related secondary carriers may provide bases for establishing evolutionary links. We anticipate that the rules governing the evolutionary expansion of ancient superfamilies will soon become more clearly defined. Such advances will undoubtedly shed light on the most basic structural restrictions that determine the propensity for functional diversification of homologous transmembrane solute-transport systems during evolution.

\section{ACKNOWLEDGEMENTS}

We wish to thank Donna Yun, Monica Mistry, Milda Simonaitis and Mary Beth Hiller for their assistance in the preparation of this manuscript. Work in the authors' laboratory was supported by NIH grants 2R01 AI14176 from The National Institute of Allergy and Infectious Diseases and 9RO1 GM55434 from the National Institute of General 
Medical Sciences, as well as by the M. H. Saier, Sr. Memorial Research Fund.

\section{REFERENCES}

Altschul, S. F., Madden, T. L., Schäffer, A. A., Zhang, J., Zhang, Z., Miller, W. \& Lipman, D. J. (1997). Gapped BLAST and PSI-BLAST : a new generation of protein database search programs. Nucleic Acids Res 25, 3389-3402.

Bateman, A., Birney, E., Durbin, R., Eddy, S. R., Howe, K. L. \& Sonnhammer, E. L. (2000). The Pfam protein families database. Nucleic Acids Res 28, 263-266.

Beckman, M. L. \& Quick, M. W. (1998). Neurotransmitter transporters: regulators of function and functional regulation. $J$ Membr Biol 164, 1-10.

Brechtel, C. E. \& King, S. C. (1998). 4-Aminobutyrate (GABA) transporters from the amino acid-polyamine-choline superfamily: substrate specificity and ligand recognition profile of the 4-aminobutyrate permease from Bacillus subtilis. Biochem J 333, 565-571.

Brechtel, C. E., Hu, L. \& King, S. C. (1996). Substrate specificity of the Escherichia coli 4-aminobutyrate carrier encoded by gabP: uptake and counterflow of structurally diverse molecules. J Biol Chem 271, 783-788.

Claros, M. G. \& von Heijne, G. (1994). TopPred II : an improved software for membrane protein structure predictions. Comput Appl Biosci 10, 685-686.

Closs, E. I., Albritton, L. M., Kim, J. W. \& Cunningham, J. M. (1993). Identification of a low affinity, high capacity transporter of cationic amino acids in mouse liver. J Biol Chem 268, 7538-7544.

Corfe, B. M., Moir, A., Popham, D. \& Setlow, P. (1994). Analysis of the expression and regulation of the gerB spore germination operon of Bacillus subtilis 168. Microbiology 140, 3079-3083.

Cosgriff, A. J. \& Pittard, A. J. (1997). A topological model for the general aromatic amino-acid permease, AroP, of Escherichia coli. J Bacteriol 179, 3317-3323.

Devereux, J., Haeberli, P. \& Smithies, O. (1984). A comprehensive set of sequence analysis programs for the VAX. Nucleic Acids Res 12, 387-395.

Devés, R. \& Boyd, C. A. R. (1998). Transporters for cationic amino acids in animal cells: discovery, structure and function. Physiol Rev 78, 487-545.

Doolittle, R. F. (1986). Of Urfs and Orfs: a Primer on How to Analyze Derived Amino Acid Sequences. Mill Valley, CA: University Science Books.

Ellis, J., Carlin, A., Steffes, C., Wu, J., Liu, J. \& Rosen, B. P. (1995). Topological analysis of the lysine-specific permease of Escherichia coli. Microbiology 141, 1927-1935.

Estévez, R., Camps, M., Rojas, A. M., Testar, X., Devés, R., Hediger, M. A., Zorzano, A. \& Palacín, M. (1998). The amino acid transport system $\mathrm{y}^{+} \mathrm{L} / 4 \mathrm{~F} 2 \mathrm{hc}$ is a heteromultimeric complex. FASEB J 12, 1319-1329.

Farcasanu, I. C., Mizunuma, M., Hirata, D. \& Miyakawa, T. (1998). Involvement of histidine permease (Hip1p) in manganese transport in Saccharomyces cerevisiae. Mol Gen Genet 259, 541-548.

Feng, D.-F. \& Doolittle, R. F. (1990). Progressive alignment and phylogenetic tree construction of protein sequences. Methods Enzymol 183, 375-387.

Hofmann, K. \& Stoffel, W. (1993). TMbase - a database of membrane spanning proteins segments. Biol Chem Hoppe-Seyler 347, 166.
Hofmann, K., Bucher, P., Falquez, L. \& Bairoch, A. (1999). The prosite database, its status in 1999. Nucleic Acids Res 27, 215-219.

Hu, L. A. \& King, S. C. (1998a). Membrane topology of the Escherichia coli $\gamma$-aminobutyrate transporter: implications on the topology and mechanism of prokaryotic and eukaryotic transporters from the APC superfamily. Biochem J 336, 69-76.

Hu, L. A. \& King, S. C. (1998b). Functional significance of the 'signature cysteine' in helix 8 of the Escherichia coli 4aminobutyrate transporter from the amino-acid-polyamine-choline superfamily. J Biol Chem 273, 20162-20167.

Hu, L. A. \& King, S. C. (1998c). Functional sensitivity of polar surfaces on transmembrane helix 8 and cytoplasmic loop 8-9 of the Escherichia coli GABA (4-aminobutyrate) transporter encoded by gabP: mutagenic analysis of a consensus amphipathic region found in transporters from bacteria to mammals. Biochem J 330, 771-776.

Isnard, A. D., Thomas, D. \& Surdin-Kerjan, Y. (1996). The study of methionine uptake in Saccharomyces cerevisiae reveals a new family of amino acid permeases. J Mol Biol 262, 473-484.

Kashiwagi, K., Shibuya, S., Tomitori, H., Kuraishi, A. \& Igaragshi, K. (1997). Excretion and uptake of putrescine by the PotE protein in Escherichia coli. J Biol Chem 272, 6318-6323.

Kim, J. W., Closs, E. I., Albritton, L. M. \& Cunningham, J. M. (1991). Transport of cationic amino acids by the mouse ecotropic retrovirus receptor. Nature 352, 725-728.

King, S. C., Fleming, S. R. \& Brechtel, C. E. (1995). Ligand recognition properties of the Escherichia coli 4-aminobutyrate transporter encoded by gabP: specificity of Gab permease for heterocyclic inhibitors. J Biol Chem 270, 19893-19897.

Kyte, J. \& Doolittle, R. F. (1982). A simple method for displaying the hydropathic character of a protein. J Mol Biol 157, 105-132.

Markovich, D., Stange, G., Bertran, J., Palacin, M., Werner, A., Biber, J. \& Murer, H. (1993). Two mRNA transcripts (rBAT-1 and rBAT-2) are involved in system $b^{0,+}$-related amino acid transport. J Biol Chem 268, 1362-1367.

Mastroberardino, L., Spindler, B., Pfeiffer, R., Skelly, P. J., Loffing, J., Shoemaker, C. B. \& Verrey, F. (1998). Amino-acid transport by heterodimers of $4 \mathrm{~F} 2 \mathrm{hc} / \mathrm{CD} 98$ and members of a permease family. Nature 395, 288-291.

Palacín, M., Estévez, R., Bertran, J. \& Zorzano, A. (1998). Molecular biology of mammalian plasma membrane amino acid transporters. Physiol Rev 78, 969-1054.

Pao, S. S., Paulsen, I. T. \& Saier, M. H., Jr (1998). The major facilitator superfamily. Microbiol Mol Biol Rev 62, 1-32.

Paulsen, I. T., Sliwinski, M. K. \& Saier, M. H., Jr (1998a). Microbial genome analyses: global comparisons of transport capabilities based on phylogenies, bioenergetics and substrate specificities. $J$ Mol Biol 277, 573-592.

Paulsen, I. T., Sliwinski, M. K., Nelissen, B., Goffeau, A. \& Saier, M. H., Jr (1998b). Unified inventory of established and putative transporters encoded within the complete genome of Saccharomyces cerevisiae. FEBS Lett 430, 116-125.

Reizer, J., Finley, K., Kakuda, D., MacLeod, C. L., Reizer, A. \& Saier, M. H., Jr (1993). Mammalian integral membrane receptors are homologous to facilitators and antiporters of yeast, fungi, and eubacteria. Protein Sci 2, 20-30.

Saier, M. H., Jr (1994). Computer-aided analyses of transport protein sequences: gleaning evidence concerning function, structure, biogenesis, and evolution. Microbiol Rev 58, 71-93.

Saier, M. H., Jr (1996). Phylogenetic approaches to the identification and characterization of protein families and superfamilies. Microb Comp Genomics 1, 129-150. 
Saier, M. H., Jr (1998). Molecular phylogeny as a basis for the classification of transport proteins from bacteria, archaea and eukarya. In Advances in Microbial Physiology, vol. 40, pp. 81-136. Edited by R. K. Poole. San Diego, CA: Academic Press.

Saier, M. H., Jr (1999a). Classification of transmembrane transport systems in living organisms. In Biomembrane Transport, pp. 265-276. Edited by L. Van Winkle. San Diego, CA: Academic Press.

Saier, M. H., Jr (1999b). Eukaryotic transmembrane solute transport systems. In International Review of Cytology: a Survey of Cell Biology, pp. 61-136. Edited by K. W. Jeon. San Diego, CA: Academic Press.

Saier, M. H., Jr (1999c). Genome archeology leading to the characterization and classification of transport proteins. Curr Opin Microbiol 2, 555-561.

Saier, M. H., Jr (2000a). Families of transmembrane transporters selective for amino acids and their derivatives. Microbiology 146, 1775-1795.

Saier, M. H., Jr (2000b). A functional/phylogenetic classification system for transmembrane solute transporters. Microbiol Mol Biol Rev 64, 354-411.

Saier, M. H., Jr, Beatty, J. T., Goffeau, A. \& 11 other authors (1999a). The major facilitator superfamily. J Mol Microbiol Biotechnol 1, 257-279.

Saier, M. H., Jr, Eng, B. H., Fard, S. \& 15 other authors (1999b). Phylogenetic characterization of novel transport protein families revealed by genome analyses. Biochim Biophys Acta 1422, 1-56.

Sanders, J. W., Leenhouts, K., Burghoorn, J., Brands, J. R., Venema, G. \& Kok, J. (1998). A chloride-inducible acid resistance mechanism in Lactococcus lactis and its regulation. Mol Microbiol 27, 299-310.

Sato, H., Tamba, M., Ishii, T. \& Bannai, S. (1999). Cloning and expression of a plasma membrane cystine/glutamate exchange transporter composed of two distinct proteins. J Biol Chem 274, 11455-11458.

Sophianopoulou, V. \& Diallinas, G. (1995). Amino acid transporters of lower eukaryotes: regulation, structure and topogenesis. FEMS Microbiol Rev 16, 53-75.

Thompson, J. D., Gibson, T. J., Plewniak, F., Jeanmougin, F. \& Higgins, D. G. (1997). The CluSTAL x windows interface: flexible strategies for multiple sequence alignment aided by quality analysis tools. Nucleic Acids Res 25, 4876-4882.

Torrents, D., Estévez, R., Pineda, M., Fernández, E., Lloberas, J., Shi, Y.-B., Zorzano, A. \& Palacín, M. (1998). Identification and characterization of a membrane protein $\left(\mathrm{y}^{+} \mathrm{L}\right.$-amino acid transporter-1) that associates with $4 \mathrm{~F} 2 \mathrm{hc}$ to encode the amino acid transport activity $\mathrm{y}^{+} \mathrm{L}$ : a candidate gene for lysinuric protein intolerance. J Biol Chem 273, 32437-32445.

Verrey, F., Jack, D. L., Paulsen, I. T., Saier, M. H., Jr \& Pfeiffer, R. (1999). New glycoprotein-associated amino acid transporters. $J$ Membr Biol 172, 181-192.

Von Heijne, G. (1992). Membrane protein structure prediction, hydrophobicity analysis and positive-inside rule. J Mol Biol 225, 487-494.

Wang, H., Kavanaugh, M. P., North, R. A. \& Kabat, D. (1991). Cell-surface receptor for ecotropic murine retroviruses is a basic amino-acid transporter. Nature 352, 729-731.

Young, G. B., Jack, D. L., Smith, D. W. \& Saier, M. H., Jr (1999). The amino acid/auxin:proton symport permease family. Biochim Biophys Acta 1415, 306-322.

Zuberi, A. R., Moir, A. \& Feavers, I. M. (1987). The nucleotide sequence and gene organization of the ger $A$ spore germination operon of Bacillus subtilis 168. Gene 51, 1-11.

Received 19 October 1999; revised 28 March 2000; accepted 24 May 2000. 